\title{
ALGORITMO LMS COM PASSO ADAPTATIVO PARA UTILIZAÇÃO EM EQUALIZADORES CEGOS BAYESIANOS
}

\author{
Alexandre Guedes de Melo e Ernesto Leite Pinto
}

\begin{abstract}
Resumo - Propõe-se um esquema de equalização cega com estimaçâo conjunta de canal e símbolos, tendo como ponto de partida dois equalizadores, apresentados em [1], com características de desempenho e complexidade computacional bem distintas. Tais características decorrem fundamentalmente dos algoritmos de filtragem adaptativa (LMS e filtro de Kalman) usados nestes equalizadores. Buscou-se um compromisso melhor entre a complexidade e o desempenho, através de uma estratégia de adaptação do tamanho de passo do algoritmo LMS com base numa métrica anteriormente utilizada para detecção cega da ocorrência de erros de equalização. Através da simulação computacional de canais com desvanecimento rápido e seletivo em freqüência é feita uma exaustiva comparaçào de desempenho entre os esquemas de equalização utilizando o LMS padrão (com passo fixo), o Filtro de Kalman e o algoritmo LMS aqui proposto. Os resultados mostram que este último produz uma melhora significativa no desempenho, em relação ao LMS padrão, e uma sensível redução na complexidade do equalizador, em relação à do esquema com filtros de Kalman.
\end{abstract}

\begin{abstract}
A blind equalizer that performs joint estimation of channel and symbols is proposed. Its origin comes from two blind equalization schemes proposed in [1] which are based on the Kalman filter and the LMS algorithm. The performance of the most complex equalizer under multipath channel models is much higher than the least complex one. A better trade-off between performance and computational complexity was searched through an investigation of strategies to improve the LMS performance. An original result obtained from this investigation is the new blind equalization algorithm here proposed which uses LMS filters with adaptive step-size parameter based on a metric used in another work for blind detection of equalization errors. An extensive performance evaluation of these equalizers under fast frequency-selective fading channels is presented. The results reached by the proposed algorithm show a remarkable improvement in the performance compared with the scheme based on standard LMS, and a reduction of the complexity compared with the one based on Kalman filters.
\end{abstract}

Palavras-chave: Equalização cega, filtro de Kalman, LMS tamanho de passo variável, comunicações móveis, complexidade computacional e análise de desempenho.

Alexandre Guedes de Melo está com a MOTOROLA - PCS Depto de Pesquisa e Desevolvimento e Emesto Leite Pinto está com o Instituto Militar de Engenharia - Depto. de Engenharia Elétrica. Endereços Eletrônicos: alexandre.g.melo@amotorola.com. ernesto@aquarius.ime.eb.br.

\section{INTRODUÇÃO}

O presente trabalho tem como objeto de interesse os sistemas de transmissão digital de portadora única em canais rádio severamente limitados em faixa e sujeitos a efeitos de multipercursos variantes no tempo, como ocorre tipicamente em diversos sistemas de comunicações móveis. Trata-se portanto de canais com desvanecimento rápido e seletivo em freqüência (DRSF).

Neste contexto, a obtenção de elevada eficiência espectral através do emprego de esquemas de modulação em fase e quadratura desempenha um papel crucial. O desempenho destes esquemas de modulação é muito sensível à interferência entre símbolos (IES) causada pelos efeitos dispersivos do canal, sendo necessário o emprego de equalizadores adaptativos para combatê-la.

As técnicas clássicas de equalização adaptativa são inicializadas por um período de treinamento baseado numa seqüência de símbolos conhecida pelo receptor. Quando o canal é variante no tempo, mesmo lentamente, a seqüência de treinamento precisa ser enviada periodicamente para evitar a ocorrência de perda de acompanhamento, reduzindo consequentemente a taxa efetiva de transmissão (vazão) de informação. Os algoritmos de equalização cega sâo de grande interesse nesse contexto, por dispensarem a transmissão de seqüências de treinamento e poderem possibilitar aumento considerável na vazão, dependendo de sua velocidade de convergência.

Dentre os vários tipos de equalizadores cegos propostos recentemente, os algoritmos com estimação conjunta de canal e símbolos, tais como os propostos por Iltis et al[1]. têm se destacado por suas promissoras características de desempenho.

No entanto, o desempenho desses equalizadores tem sido em geral avaliado com base em modelos muito simples para a resposta ao impulso do canal (RIC) invariantes no tempo, não contemplando portanto canais DRSF. Além disso, a complexidade computacional destes algoritmos, que nem sempre é devidamente avaliada, pode variar significativamente, inviabilizando em certos casos a sua utilização prática.

Desta forma, pode-se dizer que existem hoje questões em aberto sobre o desempenho desses equalizadores cegos em canais variantes no tempo e no que diz respeito ao estabelecimento de um compromisso entre complexidade e desempenho, a ponto de viabilizar sua aplicação prática num futuro próximo.

O objetivo deste trabalho é atacar estas questões e principalmente propor um novo esquema de equalização. cega com boas características de complexidade e desempenho, utilizando a simulação em computador como ferramenta para avaliação de desempenho.

O artigo está organizado como se segue. Na seção 2 são descritos os 2 algoritmos de equalização cega apresentados 
em [1]. Na seção 3 propõe-se um novo equalizador cego baseado num algoritmo LMS de passo adaptativo. Na seção 4 apresenta-se uma comparação de complexidade computacional entre esses três esquemas. A seção 5 é dedicada à avaliação do desempenho desses algoritmos de equalização, considerando-se diversos condiçōes de variabilidade da resposta ao impulso do canal. Finalmente, as conclusões são apresentadas na seção 6 .

\section{EQUALIZADORES CEGOS BAYESIA- NOS COM ESTIMAÇÃO CONJUNTA DE CANAL E SIMBOLOS}

Admite-se que a entrada do equalizador é modelada em banda básica como se mostra na Figura 1. As amostras $r(k)$ do sinal recebido são dadas por:

$$
r(k)=\sum_{m=0}^{L} b_{m}(k) d(k-m)+n(k)
$$

onde o canal tem memória $L$ e é modelado como um filtro FIR com coeficientes variantes no tempo $\left\{b_{m}(k) ; m=0, \ldots L\right\}$ e os símbolos $\{d(k)\}$ são independentes e equiprováveis, assumindo valores num conjunto de $M$ elementos. $O$ ruido $\{n(k)\} \quad$ é modelado como um processo estocástico Gaussiano complexo, estacionário em sentido amplo e branco, com distribuição de primeira ordem circularmente simétrica, de variância $\sigma_{n}{ }^{2}$.

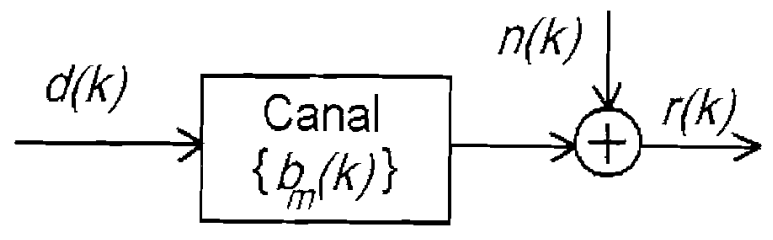

Figura 1. Modelo em Tempo Discreto do Sinal Recebido.

A Figura 2 ilustra a estrutura básica dos algoritmos propostos em [1], que realizam estimaçâo conjunta de canal e dados utilizando para o cálculo da métrica de decisão parâmetros fornecidos por um banco de $N=M^{L^{-1}}$ filtros adaptativos.

Em termos mais especificos, o papel desses filtros é gerar recursivamente estimativas condicionais da RIC associadas às $N$ possíveis subseqüências de símbolos de comprimento $L+1$. Cada uma dessas subseqüências é representada na Figura 2 por um vetor linha definido como

$$
\hat{\underline{h}}_{i}(k)=\left[d_{i}(k), d_{i}(k-1), \ldots, d_{i}(k-L)\right]
$$

Além dessas estimativas condicionais do canal, a estrutura mostrada na Figura 2 também gera como saída as probabilidades condicionais associadas a cada uma das $\mathrm{N}$ subseqüências.

A decisão é realizada com atraso e usa o critério de maximizar a probabilidade a posteriori do símbolo transmitido no instante $k$ - $L$. As probabilidades condicionais mostradas na Figura 2 são usadas no cálculo recursivo da métrica de decisão [1]. Este cálculo não é mostrado na mesma figura para fins de simplicidade.
Os dois algoritmos se diferenciam pelo tipo de filtragem adaptativa empregada. Um deles usa filtros de Kalman e por isso é denominado aqui de KF-BE (de "Kalman filter" e "blind equalizer"). Buscando uma redução significativa de complexidade, o segundo esquema usa filtros LMS e será aqui denominado LMS-BE. Deve-se notar que o cálculo de probabilidades a posteriori a rigor só é exato no esquema baseado em filtros de Kalman.

Nota-se ainda na Figura 2 que ambos os algoritmos têm uma estrutura paralela adequada para implementação em DSPs programáveis, podendo ser considerados alternativas potencialmente atraentes para aplicações práticas.

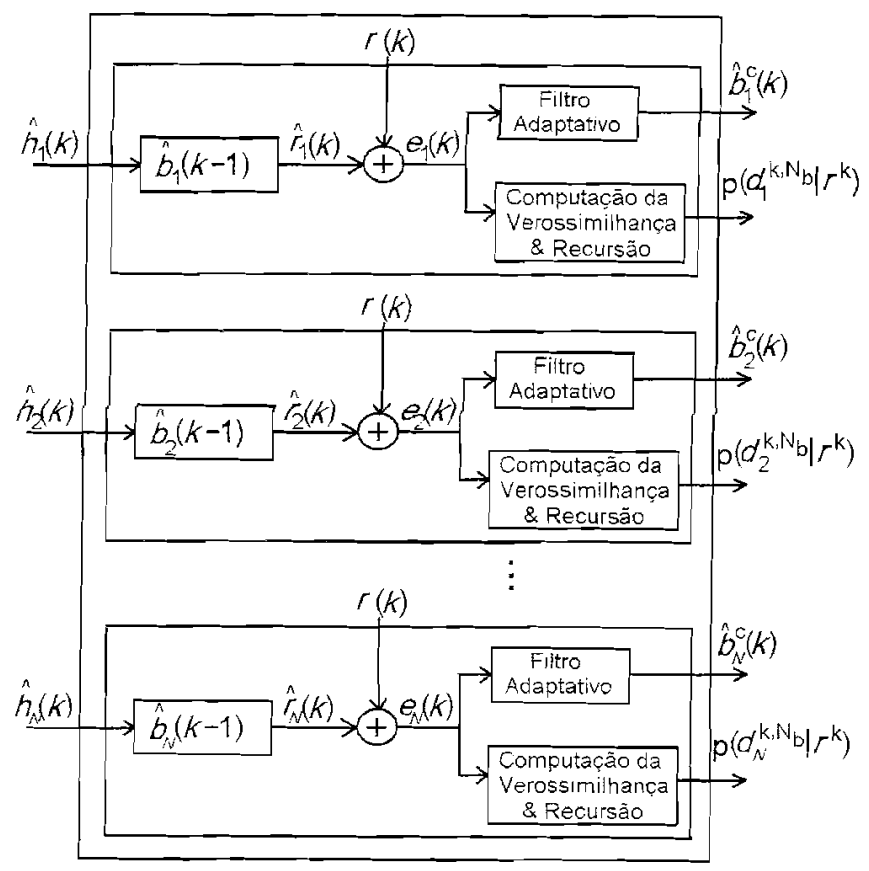

Figura 2. Equalizador Bayesiano usando Filtros Adaptativos Paralelos.

O algoritmo KF-BE está descrito em maiores detalhes na Tabela 1, onde se supõe conhecido o tamanho da memória do canal $(L)$, além da variância do ruído $\left(\sigma_{\eta 7}{ }^{2}\right)$, e a matriz de transição de estado é uma matriz identidade. As probabilidades a posteriori das subseqüèncias são calculadas recursivamente usando-se as inovações derivadas do banco de filltros de Kalman.

O equalizador LMS-BE é descrito em detalhes na Tabela 2. Este algoritmo pode ser visto como uma simplificação radical do esquema $\mathrm{KF}-\mathrm{BE}$, onde as matrizes covariância do erro de estimaçào do canal são aproximadas por uma versão escalada de uma matriz identidade e, além disso, o modelo de estado é ignorado. Como conseqüência, nenhuma operação com matrizes é necessária no esquema LMS-BE, o que o torna significativamente menos complexo que o KF$\mathrm{BE}$.

Através de avaliação do desempenho via simulação em computador de uma particular RIC invariante no tempo, Iltis et al[1] indicaram que os esquemas KF-BE e LMS-BE provêem inicialização extremamente rápida. sendo que as estimativas de canal do LMS-BE convergem mais devagar que as produzidas pelo KF-BE. Para ambos os esquemas verificou-se que a BER simulada era muito próxima daquela 
obtida admitindo-se o canal conhecido e usando o algoritmo Abend-Fritchman (decisor símbolo-a-símbolo ótimo)[2].

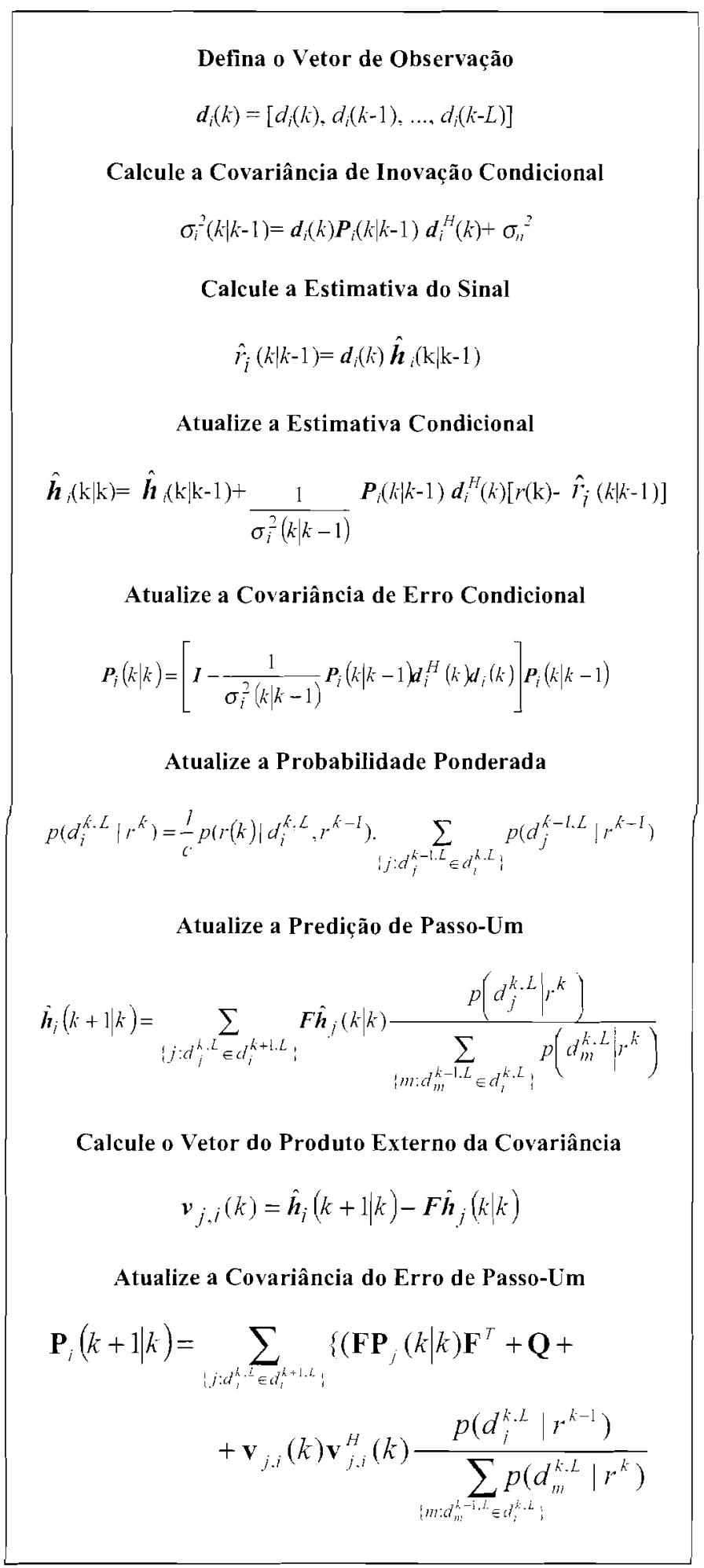

Tabela 1. Algoritmo do Equalizador KF-BE.

É importante destacar que existem duas grandes limitaçôes na avaliação do desempenho acima citada. A primeira é que os 1000 símbolos iniciais de cada realização foram descartados para efeito de avaliação da taxa de bits errados (BER, de "bit error rate"). Se o interesse pelo uso de equalizadores cegos estivesse na vazão de informação, seria provavelmente mais eficiente usar pequenas seqüências de treinamento do que descartar 1000 amostras iniciais do sinal recebido. A segunda limitação a ser ressaltada é que somente uma amostra de RIC (com coeficientes invariantes no tempo) foi utilizada em toda a avaliação apresentada.

Já em [3] a avaliação do desempenho destes algoritmos foi estendida significativamente, considerando-se canais com desvanecimento rápido e seletivo $\mathrm{em}$ freqüência modelados como GWSS-US (de "Gaussian Wide Sense Stationary - Uncorrelated Scattering"). Diferentemente do que ocorreu em [1], o desempenho de BER do esquema LMS-BE mostrou-se bem diferente daquele produzido pelo KF-BE. Em particular, verificou-se que as estimativas de canal baseadas no LMS se mostraram instáveis com muito maior freqüência do que as baseadas no KF.

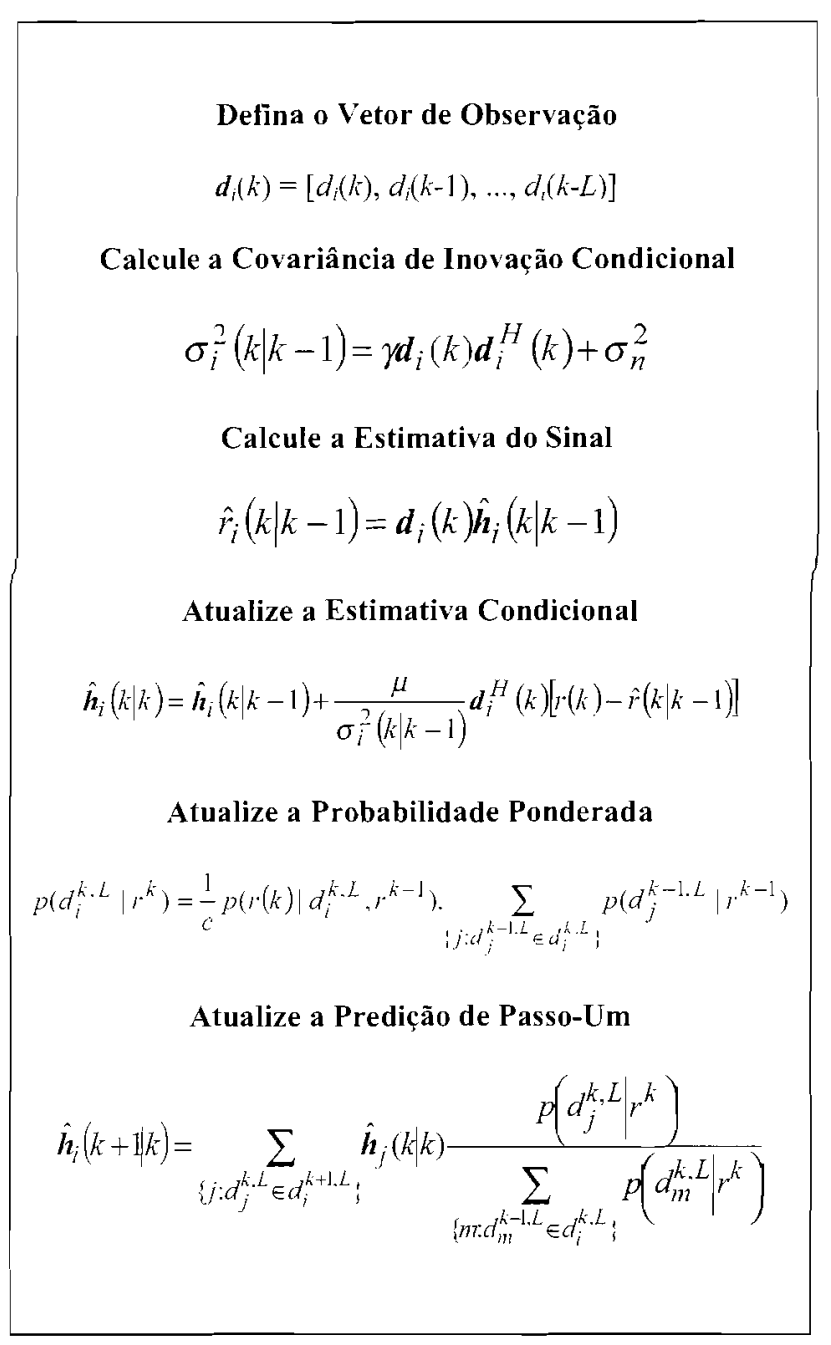

Tabela 2. Algoritmo do Equalizador LMS-BE.

A complexidade destes equalizadores é avaliada detalhadamente na seção 4, mas cabe notar de antemão que a mesma está em grande parte relacionada com a complexidade dos filtros adaptativos. Como se trata de estruturas paralelas, existe um fator nesta relação, de $M^{L+1}$. Há portanto uma grande diferença de complexidade entre os dois esquemas, sendo bastante desejável se obter um melhor compromisso entre desempenho e complexidade. Devido ao efeito multiplicativo acima mencionado, a busca de um algoritmo de filtragem adaptativa de complexidade menor é um caminho natural para se tentar alcançar tal 
compromisso. Uma proposta desta natureza é apresentada na próxima seção.

\section{ALGORITMO ASSLMS-BE}

É bem sabido que os algoritmos do tipo LMS são incapazes de satisfazer ao mesmo tempo dois requisitos conflitantes: convergência rápida e erro médio quadrático (MSE, de "Mean Square Error") pequeno. O MSE final das estimativas do LMS (admitindo a sua convergência) é diretamente proporcional ao tamanho do passo de adaptação, enquanto que o tempo de convergência aumenta à medida que o tamanho do passo diminui. Além disso, nem sempre o algoritmo LMS converge de maneira satisfatória, podendo ficar "preso" em falsos mínimos[4].

Alternativas que melhorem o desempenho do LMS têm sido propostas, procurando fazer com que o tamanho de passo assuma valores maiores no início do processamento, aumentando a taxa de convergência, e tenha valores menores à medida que o algoritmo converge, reduzindo o MSE final. Isto pode ser buscado pelo ajuste do valor do tamanho de passo de acordo com alguma indicação aproximada do estado do processo de adaptação. Vários algoritmos LMS de passo variável (VSSLMS, de "Variable Step Size Least Mean Square.") seguem esta lógica[5][6][7], utilizando-se do erro dado pela diferença entre um sinal de referência (inicialmente uma seqüência de treinamento) e a saída do filtro LMS.

No contexto de equalização cega é necessário obter um outro tipo de indicador da necessidade de se modificar o passo do algoritmo LMS. Para tanto propõe-se aqui a utilização da métrica de decisão que foi inicialmente apresentada por Dogançay e Krishnamurthy[8] para uso num algoritmo de detecção cega da ocorrência de erros de equalização. Esta métrica é apresentada a seguir.

\subsection{MÉTRICA PARA DETECÇÃO CEGA DE ERROS DE EQUALIZAÇÃO}

A Figura 3 ilustra a estrutura proposta em [8] para detecção cega da ocorrência de erros de equalização. Esta estrutura se baseia no fato (demonstrado em [9]) de que, se o canal seguir um modelo linear e invariante no tempo, a relação entre as decisôes alcançadas na saída do dispositivo de decisão e a entrada do equalizador pode ser representada pelo mesmo modelo de canal se e somente se a saida do dispositivo de decisão não contiver erros e a sequiência de entrada satisfizer uma condição de persistência de excitação[9]. Assim, é proposto em [8] um teste de hipóteses para verificar se os símbolos recebidos se ajustam ou não ao modelo de canal subjacente.

Para tanto é analisada a estimação do canal pelo algoritmo RLS ("Recursive Least Squares"), usando como entrada do modelo os símbolos decididos (saída do equalizador), como se mostra na Figura 3.

Para os objetivos do presente trabalho, o interesse recai sobre o termo de atualização do algoritmo RLS, dado por

$$
\underline{\delta}(k)=\underline{\hat{v}}(k)-\underline{\hat{v}}(k-1)
$$

onde $\hat{v}(k)$ é um vetor formado pelas estimativas dos coeficientes da RIC obtidas usando-se símbolos decididos.
Deste termo provém a métrica utilizada no teste proposto em [8], que é dada por:

$$
T(k)=\frac{\|\underline{\delta}(k)\|_{2}^{2}}{\sigma^{2} \sum_{i=1}^{P}\left(\underline{p}_{i}^{T} \hat{u}(k)\right)^{2}}
$$

onde $\underline{i}(k)$ é um vetor coluna formado por $L+l$ símbolos decididos consecutivos e $\underline{p}_{i}^{T}$ denota a i-ésima linha da inversa da matriz autocorrelação de $\underline{i}(k)$.

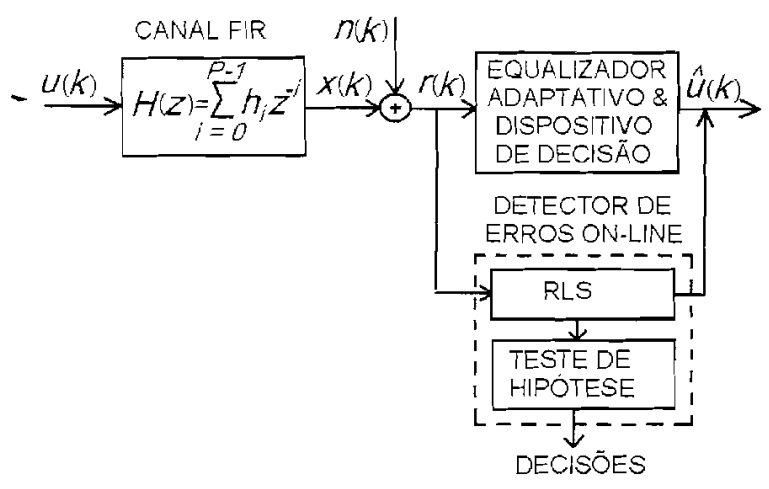

Figura 3. Estrutura de Detecção Cega de Erros de Equalização.

Mostra-se em [8] que. na ausência de erros de decisão, a distribuição de probabilidade da métrica apresentada acima é aproximadamente chi-quadrada central com um grau de liberdade (tendo portanto média 1).

Também se mostra que diante de erros de equalização a média condicional de $T(k)$ assume valores maiores. Em particular, no caso de ocorrência de um erro isolado, obtémse a seguinte aproximação para essa média:

$$
E\{T(k) \underline{P}(k), \underline{\hat{u}}(k)\}=1+\frac{h_{0}^{2} e^{2}}{\sigma_{n}^{2} \sum_{i=0}^{P}\left(\underline{p}_{i}^{T} \underline{\underline{i n}}(k)\right)^{2}}
$$

sendo $e=\frac{u(k-\Delta)}{\hat{l}(k)}-\frac{1}{\Gamma}$ e $\Gamma$ é uma constante complexa de módulo unitário associada à ambigüidade de fase intrínseca às estimativas cegas do canal.

Com base nas observações anteriores propõe-se em [8] que se decida pela ocorrência de erros se a magnitude de $T(k)$, for superior a um limiar escolhido de acordo com o valor máximo aceitável para a probabilidade de falso alarme

Cabe notar que o uso do RLS permite que as variações temporais na RIC sejam consideradas nesta mesma estratégia. desde que o próprio equalizador adaptativo seja capaz de acompanhá-las.

\subsection{UM NOVO ALGORITMO PARA AJUSTE DE PASSO EM ESQUEMAS VSSLMS}

Propõe-se a utilização da métrica descrita acima em um novo procedimento de ajuste do passo em algoritmos LMS, visto que ela provê uma medida aproximada do "sucesso" do equalizador e indiretamente reflete a evolução do 
processo de adaptação deste equalizador. Com base nas propriedades de $T(k)$, um esquema de controle apropriado para o tamanho do passo é dado por:

$$
\mu(k)=\mu_{\max }[1-\exp (-C T(k))]
$$

onde $\mu_{\max }$ é o tamanho de passo inicial e $C$ é uma constante positiva. Como pode ser visto, quando o valor de $T(k)$ aumenta, o passo $\mu(k)$ tende ao valor $\mu_{\max }$. Em caso contrário $\mu(k)$ é reduzido.

$\mathrm{Na}$ equação (6) existem 2 (duas) constantes, $\mu_{\text {max }}$ e $C$, a serem determinadas. A primeira pode ser obtida por um processo de otimização via simulação, de forma similar ao que geralmente é feito no caso do algoritmo LMS de passo fixo. Uma escolha criteriosa do valor da constante $C$ requer o conhecimento de parâmetros estatísticos da métrica $T(k)$, tanto na ausência quanto na presença de erros de equalização. No entanto, a obtenção de tais parâmetros na presença de erros de equalização é extremamente complexa

Pode-se notar expressão (5) que a magnitude do aumento na média condicional de $T(k)$ diante da ocorrência de um erro de equalização é determinada pelo segundo termo do lado direito, que é significativamente afetado pelo valor de $\sigma_{\mathrm{n}}{ }^{2}$. O peso de $\sigma_{\mathrm{n}}{ }^{2}$ tende a minimizar a influência do erro de equalização propriamente dito no valor desta média, o que não é desejável para o controle do passo.

Visando contornar este problema, propõe-se aqui o uso de dois valores distintos para $C$, denominados $C_{1}$ e $C_{2}$ $\left(\mathrm{C}_{1}>\mathrm{C}_{2}\right)$, com o intuito de que, na situação em que erros de equalização sejam detectados, $C_{1}$ enfatize na métrica $T(k)$ o peso destes erros (permitindo o aumento do passo) e, na situação oposta, $C_{2}$ leve a valores pequenos de passo.

Pela discussão acima, surge a necessidade de se estabelecer uma comparação com um limiar $\eta$ para se decidir quando utilizar $\mathrm{C}_{1}$ ou $\mathrm{C}_{2}$ (em lugar de $\mathrm{C}$ ) na equação (6). O critérjo aqui adotado para determinação desse limiar é o mesmo empregado em [8] na detecção da ocorrência de erros de equalização, sendo baseado no valor máximo aceitável para a probabilidade de falso alarme.

Alguns testes iniciais revelaram que, mesmo com a utilização de um passo adaptativo, o equalizador baseado no algoritmo VSSLMS pode ficar perdido em falsos mínimos, não convergindo para a RIC.

Este fato motivou a busca de outro melhoramento no algoritmo proposto, que é a utilização da métrica também como um critério para se decidir pelo reinício da estimação do canal, em caso do mesmo não parecer estar convergindo. Desta forma, escolheu-se um limiar de detecçào de divergência $\eta_{\text {div }}$ associado ao valor de $T(k)$. e um contador do número de vezes que este limiar é ultrapassado. Se este contador alcançar um determinado patamar, admite-se que o algoritmo ainda não convergiu, provavelmente por estar num falso mínimo, e deve ser reiniciado.

A Figura 4 apresenta um exemplo de realização da métrica $T(k)$, para ilustrar diferentes situações relativas ao ajuste do passo do LMS com base nesta métrica. Três regiões distintas são identificadas. Na região 3 , em que $T(k)<\eta$, é provável a ausência de erros de equalização e por isso a constante $\mathrm{C}_{2}$ deve ser utilizada em (6). Na região 2 , em que $\eta_{\text {di }}<T(k)<\eta$. é a provável ocorrência de erros de equalização. o que leva à necessidade de se utilizar a constante $C_{1}$. A região 1 , em que $T(k)>\eta_{\text {div }}$, refere-se à situação em que o contador de ultrapassagens do limiar de detecção de divergência $\eta_{\mathrm{div}}$ deve ser incrementado, além de se usar a constante $C_{1}$.

\begin{tabular}{|c|c|c|c|}
\hline \begin{tabular}{|l|} 
Variável \\
\end{tabular} & Somas & Multiplicações & Div. \\
\hline$\sigma_{\mathrm{i}}^{2}(\mathrm{k})$ & $N \cdot(L+1) \cdot(L+1)$ & $N \cdot(L+1) \cdot(L+2)$ & 0 \\
\hline$\hat{r_{i}}(k \mid k)$ & N.L & $N .(L+1)$ & 0 \\
\hline$\hat{h}_{i}(k \mid k)$ & $N \cdot[(L+1) \cdot(L+1)+1]$ & $N \cdot(L+1) \cdot(L+2)$ & $\mathrm{N}$ \\
\hline $\mathbf{P}_{\mathrm{i}}(\mathrm{k} \mid \mathrm{k})$ & $\begin{array}{l}N .(L+1)[(L+1) . \\
(L+1)+L]\end{array}$ & $\begin{array}{l}N .(L+1) \cdot(L+1) . \\
(L+4)\end{array}$ & $\mathrm{N}$ \\
\hline$p\left(d^{k . L} \cdot r^{k}\right)$ & $N \cdot(11+M)-1$ & $33 N$ & $\mathrm{~N}$ \\
\hline$\hat{\boldsymbol{h}_{i}}(k+1 \mid k)$ & $\begin{array}{l}(N / M) \cdot[(M-1) \\
(L+2)+L \cdot(L+1)]\end{array}$ & $\begin{array}{l}(N / M) \cdot(L+1) \cdot[2 M \\
+(L+1)]\end{array}$ & $\mathrm{N} / \mathrm{M}$ \\
\hline$v_{i . i}$ & N.M. $(L+1)(L+1)$ & N.M. $(L+1)(L+1)$ & 0 \\
\hline $\boldsymbol{P}_{i}(k+1 \mid k)$ & $\begin{array}{l}(N / M) \cdot 2 \cdot(L+1) \\
(L+1) \cdot(L+1) \cdot(M-1)\end{array}$ & $\begin{array}{l}(N / M) \cdot 2 \cdot M \cdot(L+1) \\
.(L+1) \cdot(L+2)\end{array}$ & $\mathrm{N} / \mathrm{M}$ \\
\hline
\end{tabular}

Tabela 3. Contagem de instruçòes por amostra para o equalizador KF-BE.

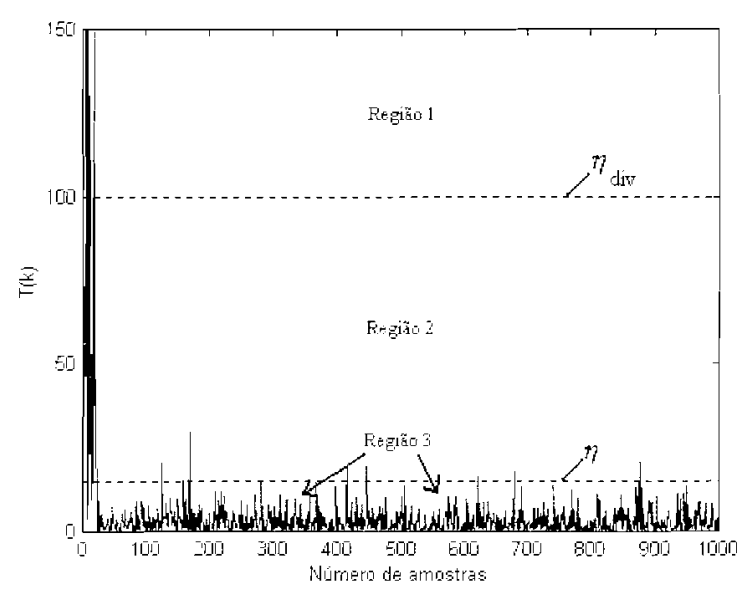

Figura 4. Exemplo de evolução da métrica $T(k)$.

Utilizando-se este algoritmo LMS de passo adaptativo para obtenção das estimativas condicionais de canal no algoritmo descrito na Figura 2 e na Tabela 2 obtém-se um novo equalizador cego Bayesiano, doravante denominado ASSLMS-BE. Do exposto acima, vê-se que há 5 (cinco) parâmetros a serem fixados para a utilização deste equalizador: $C_{1}, C_{2}, \eta, \eta_{\mathrm{di} \text { i }}$ e o limite do contador de ultrapassagens do limiar $\eta_{\text {div. Nas simulações cujos }}$ resultados são apresentados na seção 5, os valores destas constantes foram fixados de forma empírica, levando-se em conta fatores explicados a seguir

O valor escolhido para $\eta$ foi 15 , o que leva a uma probabilidade de falso alarme menor que $0,5 \%[8]$. Testes iniciais revelaram que, tanto para $\eta_{\text {div }}$ quanto para o limite do seu contador de ultrapassagem, a escolha do valor 100 leva a resultados satisfatórios no que se refere à indicação da necessidade de se reinicializar o algoritmo pela provável não convergência. Outros testes mostraram ser satisfatória a escolha do valor de $C_{1}$ igual a $\sigma_{n}{ }^{2}$, de forma a compensar a influência da variância do ruído sobre $T(k)$. Por outro lado, 
a escolha do valor da constante $C_{2}$ igual a 0,1 permite que seja alcançado um valor de passo pequeno e, em conseqüência, menores valores de MSE residual.

\section{COMPLEXIDADE COMPUTACIONAL}

Na implementação digital das etapas de banda básica de transceptores geralmente é utilizada uma divisão das funções entre DSPs e Microcontroladores. A escolha é dirigida pelas restrições de tempo de desenvolvimento, custo, taxas de transmissão impostas, entre outros requisitos de sistema. Baseado nestes fatores, a opção de se implementar em DSP a função de equalização tem sido comumente adotada, particularmente no caso de comunicações móveis.

Como a equalização é reconhecidamente uma das funções que mais exigem recursos do DSP, procura-se a seguir avaliar com atenção a complexidade dos três algoritmos investigados neste trabalho.

Para tanto foi avaliado o número de ciclos de instrução do processador requeridos pelos algoritmos KF-BE, LMSBE e ASSLMS-BE para cada amostra de entrada. As Tabelas 3, 4 e 5 contêm o número aproximado de operações de soma, multiplicação e divisão em função de $N$ (número de subseqüências $\boldsymbol{d}_{i}^{h . L}$ ), $M$ (tamanho do alfabeto de símbolos), e $L$ (comprimento da memória do canal), para cada um dos algoritmos. É importante lembrar que se admite o conhecimento a priori de $L$ e de $\sigma_{n}{ }^{2}$. Considera-se que o cálculo da função exponencial envolve 32 instruções de multiplicação e 11 instruções de soma [11].

\begin{tabular}{|c|c|c|c|}
\hline Variável & Somas & Multiplicações & Div. \\
\hline$\hat{r}_{i}(k \mid k)$ & N.L & $N .(L+1)$ & 0 \\
\hline$\hat{\boldsymbol{h}}_{i}(k \mid k)$ & $N .(L+2)$ & $N .(L+2)$ & 0 \\
\hline $\mathrm{p}\left(\mathrm{d}_{\mathrm{i}}^{\mathrm{k} . \mathrm{L}} \mid \mathrm{r}^{\mathrm{k}}\right)$ & $N .(11+M)-1$ & $33 N$ & $\mathrm{~N}$ \\
\hline$\hat{\boldsymbol{h}}_{i}(k+1 \mid k)$ & $\begin{array}{c}(N / M) \cdot(M-1) . \\
(L+2)\end{array}$ & $(N / M) \cdot 2 M .(L+1)$ & $\mathrm{N} / \mathrm{M}$ \\
\hline
\end{tabular}

Tabela 4. Contagem de instruções por amostra para o equalizador LMS-BE.

\begin{tabular}{|l|l|l|l|}
\hline Variável & Somas & Multiplicações & Div. \\
\hline$\hat{r}_{i}(k \mid k)$ & N.L & $N .(L+1)$ & 0 \\
\hline $\boldsymbol{h}_{i}(k \mid k)$ & $N .(L+2)$ & $N .(L+2)$ & 0 \\
\hline $\mathrm{p}\left(\mathbf{d}_{\mathrm{i}}{ }^{\mathrm{k} . \mathrm{L}} \mathbf{r}^{\mathbf{k}}\right)$ & $N .(11+M)-1$ & $33 N$ & $\mathrm{~N}$ \\
\hline$\hat{\boldsymbol{h}}_{i}(k+1 k)$ & $(N / M) .(M-1) \cdot(L+2)$ & $(N / M) \cdot 2 M .(L+1)$ & $\mathrm{N} / \mathrm{M}$ \\
\hline $\mathbf{W}(\mathrm{k})$ & $(L+1) .(2 L+1)$ & $(L+1)(2 L+5)+1$ & 3 \\
\hline$\xi(k)$ & $L+1$ & $L+1$ & 0 \\
\hline$\hat{\boldsymbol{h}}(k)$ & $L+1$ & $L+1$ & 0 \\
\hline $\boldsymbol{P}(k)$ & $(L+1) .(L+1) .(L+1)$ & $(L+1) \cdot[(L+1)$. & 2 \\
& & $(L+1)+2 L+3]$ & \\
\hline $\boldsymbol{T}(k)$ & $L .(L+1)+3 L+1$ & $(L+1)^{2}+2 L+3$ & 1 \\
\hline$\mu(\mathrm{k})$ & 12 & 34 & 0 \\
\hline
\end{tabular}

Tabela 5. Contagem de instruções por amostra para o equalizador ASSLMS-BE.
Com a finalidade de computar o número de instruções necessárias por segundo, foram feitas as seguintes suposições, que são consistentes com a maioria dos dispositivos DSP:

- Uma multiplicação e uma soma são realizadas em um ciclo de instrução [10];

- Uma divisão requer 24 ciclos de instrução. (Este numero é dependente do processador utilizado; 24 se aplica ao DSP Motorola 56000[11]).

valor de taxa de símbolos foi fixado em 24,3 Kbaud, que é um valor típico de sistemas de comunicações móveis de segunda geraçâo. Considerou-se ainda $L=2$.

$\mathrm{Na}$ Figura 5 são apresentadas as complexidades computacionais dos algoritmos assim obtidas. Esta Figura mostra claramente a alta complexidade alcançada quando se usa modulação QPSK. Em termos de comparação entre equalizadores distintos, vê-se que a complexidade do LMS-

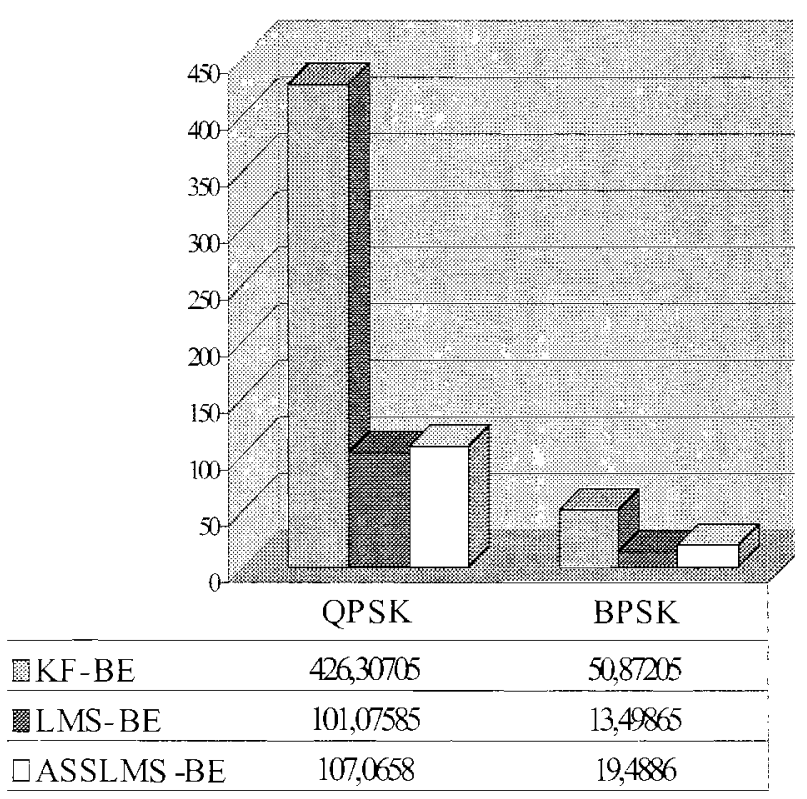

Figura 5. Comparação da Complexidade Computacional (instruções por amostra) dos Algoritmos KF-BE, LMSBE e ASSLMS-BE.

$\mathrm{BE}$ é cerca de 4 vezes menor do que a do $\mathrm{KF}-\mathrm{BE}$ para os dois tipos de modulação. Já a do ASSLMS-BE é próxima a do LMS-BE, sendo maior em aproximadamente $45 \%$ para modulação BPSK e em cerca de 5\% para modulação QPSK.

A maior proximidade entre as complexidades computacionais do ASSLMS-BE e do LMS-BE para a modulação QPSK se deve ao fato de que os cálculos adicionais do primeiro em relação ao segundo não estão relacionados nem com o tamanho de alfabeto de símbolos $(\mathrm{M})$, nem com o número de subseqüências $(\mathrm{N})$.

Em síntese, pode-se dizer que o esquema ASSLMS aqui proposto efetivamente apresenta boas características de complexidade computacional quando comparado aos outros dois algoritmos.

\section{AVALIAÇÃO DE DESEMPENHO}

Várias simulações foram realizadas com o objetivo de avaliar o desempenho do equalizador ASSLMS-BE. Para 
efeito de comparação, também se avaliou o desempenho obtido dos esquemas KF-BE e LMS-BE. Em todas as simulações foi utilizada modulação BPSK.

Utilizou-se como principais indicadores de desempenho a taxa de bits errados e o erro médio quadrático na estimação do canal. As estimativas de todos os parâmetros de desempenho foram obtidas através de médias empíricas calculadas a partir de 250 realizações independentes de todos os processos estocásticos envolvidos na transmissão de 1000 bits, o que perfaz um total de 250.000 bits.

\subsection{MODELAGEM E SIMULAÇÃO DO CANAL}

Adotou-se o seguinte modelo equivalente em banda básica para resposta ao impulso do canal:

$$
h(t, \tau)=G_{0}(t) \delta(t)+G_{1}(t) \delta(t-T)+G_{2}(t) \delta(t-2 T)
$$

onde $T$ é a duração do intervalo de símbolo e os ganhos de derivação $G_{0}(t), G_{l}(t)$ e $G_{2}(t)$, também denominados coeficientes do canal, são processos estocásticos Gaussianos complexos, estacionários, de média nula, independentes entre si, com distribuição de amplitude de Rayleigh e distribuição de fase uniforme.

A densidade espectral de potência de cada um desses processos está associada ao modelo de espalhamento Doppler adotado e é dada por

$$
S_{i}(f)=\left\{\begin{array}{cc}
\frac{C_{i}(0)}{2 \pi \sqrt{f_{d}^{2}-f^{2}}}, & |f|<f_{d} \\
0, & |f|>f_{d}
\end{array}\right.
$$

onde $f_{d}$ é o deslocamento Doppler máximo, dado por:

$$
f_{d}=\left(\frac{v}{c}\right) f_{c}
$$

sendo $\mathrm{v}$ a velocidade relativa entre transmissor e receptor, $\mathrm{c}$ a velocidade da luz no vácuo e $f_{c}$ a freqüência da portadora.

$O$ formato de Espectro Doppler contido em (8), conhecido como modelo de Jakes, tem sido freqüentemente utilizado no desenvolvimento de simuladores de canais rádio móveis. particularmente em ambientes urbanos. Este modelo foi adotado nos Estados Unidos pela TIA ("Telecommunications Industry Association") para avaliação de desempenho do padrão IS-136 [12] de telefonia móvel digital e também para avaliação de alguns serviços previstos no padrão de terceira geração UWC 136.

As variâncias dos ganhos da primeira, segunda e terceira derivações do modelo de canal foram fixadas em $0,5,0,3 \mathrm{e}$ 0,2 , respectivamente. Para simulação deste modelo utilizouse o método de Monte-Carlo, por ter se mostrado excelentes características de desempenho em um trabalho anterior dedicado à avaliação de técnicas para simulação de canais GWSS-US [13].

Essa técnica de simulação reflete diretamente a estrutura de múltiplos percursos do canal. A geração de uma amostra da resposta o impulso do canal é dada pela superposição linear de exponenciais complexas que representam o efeito de percursos elementares individualmente caracterizados pelos valores de atraso $\tau_{n}$, deslocamento Doppler $v_{n}$ e amplitude complexa $A_{n}$.
Cada ganho de derivação simulado pela técnica de Monte Carlo pode ser dado por:

$$
G_{i}(t)=\sqrt{\frac{\sigma_{i}^{2}}{N_{C}}} \sum_{j=1}^{N_{C}} A_{j i} e^{j 2 \pi v_{j i} t}
$$

onde $\sigma_{i}^{2}$ é a variância do ganho, $\left\{A_{j i}\right\}$ são variáveis aleatórias Gaussianas complexas, independentes, de média nula e variância unitária, e $\left\{v_{j i}\right\}$ são variáveis aleatórias independentes e identicamente distribuídas, com função densidade de probabilidade igual ao espectro Doppler normalizado do ganho de derivação. O parâmetro $N_{C}$ é denominado ordem do modelo e foi fixado em 20 no presente trabalho, com base em resultados obtidos em [13].

Para o modelo de Jakes aqui adotado, as variáveis aleatórias $\left\{v_{j i}\right\}$ podem ser obtidas aplicando-se a seguinte transformação:

$$
V_{i i}=\mathrm{g}\left(\mathrm{w}_{\mathrm{j}}\right)=\mathrm{f}_{\mathrm{D}} \cdot \cos \left(2 \pi \cdot \mathrm{w}_{\mathrm{j}}\right) \quad 0 \leq \mathrm{w}_{\mathrm{j}} \leq 1
$$

onde $w_{j}$ e uma variável aleatória com distribuição uniforme em $[0,1\}$.

Para a avaliação de algoritmos LMS de passo fixo é necessário estabelecer o tamanho do passo. No caso do algoritmo ASSLMS-BE é necessário estabelecer o valor máximo permitido para o passo. Esta determinação foi feita numericamente, procurando-se minimizar a BER estimada, para cada valor admitido para a razão $E_{h} / N_{0}$ na entrada do receptor, sendo $E_{b}$ a energia por bit e $N_{0}$ o valor da densidade espectral unilateral de potência do ruido.

Para o estabelecimento da equação de estado usada pelo algoritmo KF-BE, utilizou-se um modelo de passeio aleatório para representar a evolução dos coeficientes da RIC com o tempo. A variância do ruído deste modelo foi ajustada de acordo com a variação na função autocorrelação dos coeficientes da RIC em um intervalo de símbolo, a qual depende do valor do deslocamento Doppler máximo, $f_{D}$.

\subsection{CANAL INVARIANTE NO TEMPO}

Considerou-se inicialmente que os coeficientes do canal são invariantes no tempo, o que corresponde a admitir $f_{\mathrm{D}}=$ 0. A Tabela 6 apresenta os valores obtidos para o passo do algoritmo LMS-BE e para o passo máximo do ASSLMS$\mathrm{BE}$, através do procedimento numérico de otimização descrito anteriormente.

As curvas de taxa de erro em função de $E_{h} / N_{0}$ produzidas pelos 3 equalizadores são mostradas na Figura 6. Os algoritmos apresentam desempenhos próximos para valores de $E_{b} / N_{0}$ abaixo de $10 \mathrm{~dB}$, aproximadamente. A partir deste valor, e até $40 \mathrm{~dB}$, o desempenho do algoritmo LMS-BE praticamente não se altera, enquanto que o desempenho do KF-BE continua melhorando. Já o desempenho do algoritmo ASSLMS-BE fica aproximadamente inalterado entre $15 \mathrm{~dB}$ e $25 \mathrm{~dB}$, voltando a melhorar a taxas equivalentes à do KF-BE a partir dos $25 \mathrm{~dB}$. O comportamento entre $15 \mathrm{~dB}$ e $25 \mathrm{~dB}$ pode ser explicado pelo esquema de reinicialização que, numa situação de divergência das estimativas da RIC (para uma mesma realização de canal e seqüência transmitida), pode ser acionado mais cedo no caso em que a razão $E_{b} / N_{0}$ é menor. 
Isto explica também o fato do desempenho obtido com $E_{b} / N_{0}=15 \mathrm{~dB}$ ser melhor do que o obtido com $E_{b} / N_{0}=20 \mathrm{~dB}$.

\begin{tabular}{|l|c|c|l|l|l|l|l|l|l|}
\hline $\mathrm{E}_{\mathrm{b}} / \mathrm{N}_{0}$ & $\begin{array}{c}0 \\
\end{array}$ & $\begin{array}{c}5 \\
\mathrm{~dB}\end{array}$ & $\begin{array}{l}10 \\
\mathrm{~dB}\end{array}$ & $\begin{array}{l}15 \\
\mathrm{~dB}\end{array}$ & $\begin{array}{l}20 \\
\mathrm{~dB}\end{array}$ & $\begin{array}{l}25 \\
\mathrm{~dB}\end{array}$ & $\begin{array}{l}30 \\
\mathrm{~dB}\end{array}$ & $\begin{array}{l}35 \\
\mathrm{~dB}\end{array}$ & $\begin{array}{l}40 \\
\mathrm{~dB}\end{array}$ \\
\hline LMS & 0,05 & 0.15 & 0.20 & 0.30 & 0,30 & 0.35 & 0.35 & 0.35 & 0.35 \\
\hline ASS & 0.05 & 0.25 & 0.65 & 0.85 & 0.55 & 0.80 & 0.80 & 0.70 & 0.70 \\
\hline
\end{tabular}

Tabela 6. Valores de passo do LMS-BE e de passo máximo do ASSLMS-BE para canal invariante no tempo.

A Figura 6 mostra a existência de pisos nas curvas de desempenho dos 3 equalizadores, sendo alcançado rapidamente para o algoritmo LMS-BE e de forma mais lenta para os outros dois. Estes pisos decorrem principalmente de erros que ocorrem intensamente antes que se alcance a convergência nas estimativas de canal também e em casos de divergência dos algoritmos. Cabe aqui lembrar que todos os bits recebidos são levados em conta na avaliação da taxa de erro, inclusive os detectados no início do processamento, quando o equalizador desconhece completamente a RIC.

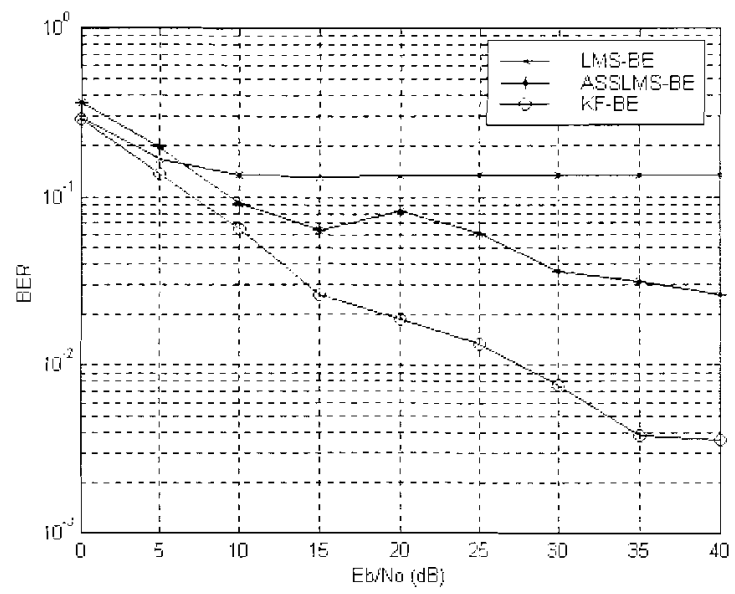

Figura 6. Curvas de Desempenho de BER para canal invariante no tempo.

Uma visualização da distribuição dos erros de decisão ao longo do tempo pode ser obtida subdividindo os blocos de bits detectados em grupos de mesmo tamanho e avaliando a evolução da porcentagem de bits errados por grupos.

A Figura 7 foi obtida desta forma, sendo os grupos formados de 100 bits. Cada valor de porcentagem de bits errados foi portanto obtido com base em 25000 bits detectados, originados de 250 simulações independentes. Esta Figura mostra que a quantidade de erros durante a inicializaçào do equalizador é muito grande, tanto para o LMS-BE quanto para o ASSLMS-BE. Também se nota na Figura 7 que para o LMS-BE esta quantidade de erros permanece na mesma faixa de valores durante todo o processamento de recepção, enquanto que para o ASSLMS$\mathrm{BE}$ e para o KF-BE existe uma queda acentuada com o tempo. Isto mostra a capacidade destes dois últimos algoritmos de melhorar as estimativas de símbolos ao longo do processamento de cada bloco de amostras recebidas.

A Figura 8 apresenta os resultados de MSE produzidos por cada um dos algoritmos na estimação da RIC. Pode ser visto que o KF-BE apresenta a taxa de convergência mais rápida e também alcança o menor valor de MSE residual. Exatamente o oposto acontece com o LMS-BE: além de apresentar uma convergência lenta, apresenta um elevado valor para o MSE residual. O desempenho do ASSLMS-BE fica entre os desempenhos dos outros dois algoritmos, porém muito mais próximo ao do KF-BE, tanto no que se refere à taxa de convergência quanto ao MSE residual.

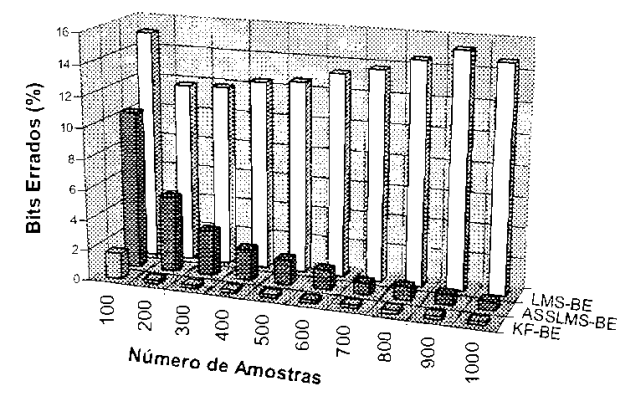

\begin{tabular}{lc|ccccccc|c|c|} 
& 100 & 200 & 300 & 400 & 500 & 600 & 700 & 800 & 900 & 1000 \\
\hline KF-BE & 1,72 & 0,212 & 0,188 & 0,208 & 0.2 & 0,184 & 0,216 & 0,264 & 0,212 & 0,212 \\
\hline ASSLMS-BE & 10,388 & 4,988 & 2,996 & 2,024 & 1,644 & 1,268 & 0,768 & 0,824 & 0,672 & 0,56 \\
\hline LMS-BE & 15,096 & 11,776 & 11,856 & 12,408 & 12,616 & 13,32 & 13,764 & 14,556 & 15,304 & 14,74 \\
\hline
\end{tabular}

Figura 7. Porcentagem de bits errados ao longo dos blocos de símbolos recebidos: canal invariante no tempo e $\mathrm{E}_{\mathrm{b}} / \mathrm{N}_{0}=40 \mathrm{~dB}$.

Para ilustrar o efeito do mecanismo de reinicialização, escolheu-se uma realização específica da RIC para a qual os algoritmos LMS-BE e ASSLMS-BE inicialmente não convergem. como pode ser visto na Figura 9. Nota-se nesta Figura que por volta da amostra 200 o algoritmo ASSLMS$\mathrm{BE}$ é reinicializado (o contador de divergência ultrapassa o respectivo limiar) e a partir daí consegue sair de uma condição de falso mínimo, melhorando significativamente as estimativas da RIC, a ponto de apresentar erro de estimação próximo do produzido pelo esquema KF-BE a partir da amostra 500, aproximadamente. Já o algoritmo LMS-BE permanece na condição de falso mínimo durante toda a realizaçào mostrada na Figura 9.

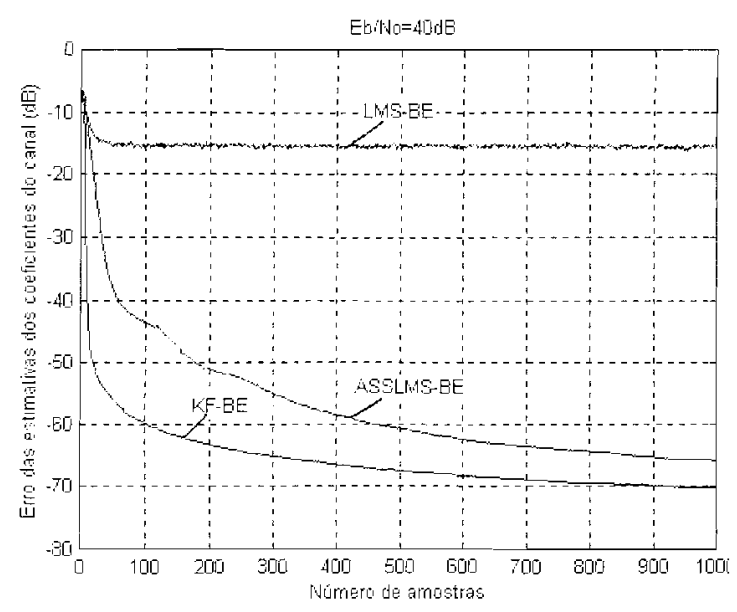

Figura 8. Trajetória de EMQ na estimação dos coeficientes do canal invariante no tempo. 


\subsection{CANAL VARIANTE NO TEMPO}

A fim de avaliar o desempenho dos equalizadores diante de variações da RIC com o tempo, utilizou-se os valores de 10,50 e $100 \mathrm{~Hz}$ para o deslocamento Doppler máximo, fixando-se a taxa de simbolos em 24,3 Kbaud.

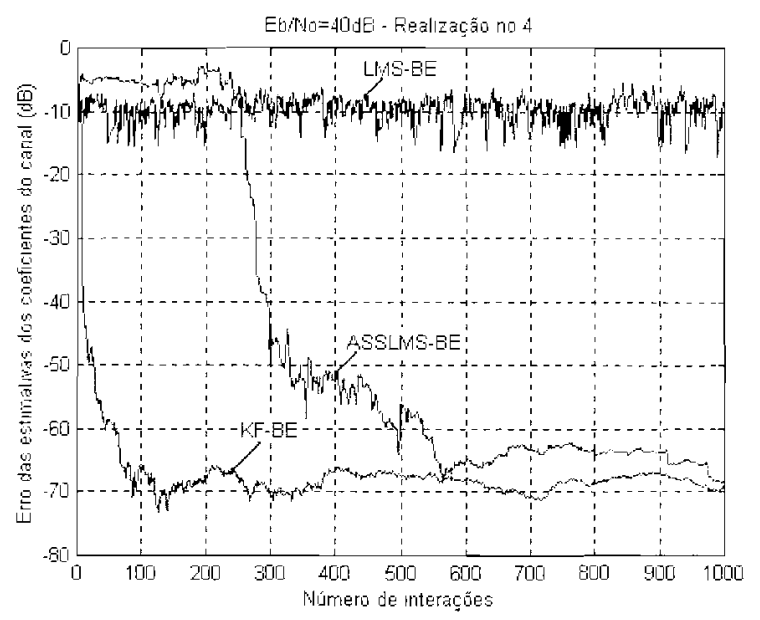

Figura 9. Exemplo do efeito da reinicialização sobre o algoritmo ASSLMS-BE.

As Tabelas 7,8 e 9 apresentam os resultados de otimização numérica do passo do LMS-BE e do passo máximo do ASSLMS-BE para cada um dos valores de $f_{\mathrm{D}}$ admitidos.

\begin{tabular}{|l|l|l|l|l|l|l|l|l|l|}
\hline $\mathbf{E}_{\mathbf{b}} / \mathbf{N}_{0}$ & $\begin{array}{c}0 \\
\mathrm{~dB}\end{array}$ & $\begin{array}{c}5 \\
\mathrm{~dB}\end{array}$ & $\begin{array}{l}10 \\
\mathrm{~dB}\end{array}$ & $\begin{array}{l}15 \\
\mathrm{~dB}\end{array}$ & $\begin{array}{l}20 \\
\mathrm{~dB}\end{array}$ & $\begin{array}{l}25 \\
\mathrm{~dB}\end{array}$ & $\begin{array}{l}30 \\
\mathrm{~dB}\end{array}$ & $\begin{array}{l}35 \\
\mathrm{~dB}\end{array}$ & $\begin{array}{l}40 \\
\mathrm{~dB}\end{array}$ \\
\hline $\mathbf{L M S}$ & 0.05 & 0.15 & 0.20 & 0,20 & 0.25 & 0.25 & 0.25 & 0.25 & 0.25 \\
\hline $\mathbf{A S S}$ & 0.10 & 0.20 & 0.45 & 0,80 & 0,90 & 0.90 & 0.80 & 0.70 & 0,70 \\
\hline
\end{tabular}

Tabela 7. Valores de passo do LMS-BE e de passo máximo do ASSLMS-BE para canal variante no tempo $-f_{D}=10 \mathrm{~Hz}$.

\begin{tabular}{|l|l|l|l|l|l|l|l|l|l|}
\hline $\mathbf{E}_{\mathbf{b}} / \mathbf{N}_{\mathbf{u}}$ & $\begin{array}{c}0 \\
\mathrm{~dB}\end{array}$ & $\begin{array}{l}\mathrm{dB} \\
\mathrm{dB}\end{array}$ & $\begin{array}{l}10 \\
\mathrm{~dB}\end{array}$ & $\begin{array}{l}15 \\
\mathrm{~dB}\end{array}$ & $\begin{array}{l}20 \\
\mathrm{~dB}\end{array}$ & $\begin{array}{l}25 \\
\mathrm{~dB}\end{array}$ & $\begin{array}{l}30 \\
\mathrm{~dB}\end{array}$ & $\begin{array}{l}35 \\
\mathrm{~dB}\end{array}$ & $\begin{array}{l}40 \\
\mathrm{~dB}\end{array}$ \\
\hline $\mathbf{L M S}$ & 0.10 & 0.15 & 0.15 & 0.15 & 0,20 & 0.15 & 0.20 & 0.20 & 0.20 \\
\hline $\mathbf{A S S}$ & 0.30 & 0.40 & 0.55 & 0.75 & 0.85 & 0.85 & 0.85 & 0.90 & 0.70 \\
\hline
\end{tabular}

Tabela 8. Valores de passo do LMS-BE e de passo máximo do ASSLMS-BE para canal variante no tempo $-f_{D}=50 \mathrm{~Hz}$.

\begin{tabular}{|l|l|l|l|l|l|l|l|l|l|}
\hline $\mathbf{E}_{\mathfrak{h}} / \mathbf{N}_{0}$ & $\begin{array}{c}0 \\
\mathrm{~dB}\end{array}$ & $\begin{array}{l}5 \\
\mathrm{~dB}\end{array}$ & $\begin{array}{l}10 \\
\mathrm{~dB}\end{array}$ & $\begin{array}{l}15 \\
\mathrm{~dB}\end{array}$ & $\begin{array}{l}20 \\
\mathrm{~dB}\end{array}$ & $\begin{array}{l}25 \\
\mathrm{~dB}\end{array}$ & $\begin{array}{l}30 \\
\mathrm{~dB}\end{array}$ & $\begin{array}{l}35 \\
\mathrm{~dB}\end{array}$ & $\begin{array}{l}40 \\
\mathrm{~dB}\end{array}$ \\
\hline LMS & 0.15 & 0,15 & 0,15 & 0.20 & 0.20 & 0.20 & 0.20 & 0.20 & 0.20 \\
\hline ASS & 0.40 & 0.45 & 0.50 & 0.75 & 0.75 & 0.70 & 0.75 & 0.70 & 0.70 \\
\hline
\end{tabular}

Tabela 9. Valores de passo do LMS-BE e de passo máximo do ASSLMS-BE para canal variante no tempo $-f_{\mathrm{D}}=100 \mathrm{~Hz}$.

As curvas de BER obtidas com deslocamento Doppler máximo de $10 \mathrm{~Hz}$ são apresentadas na Figura 10. Como no caso do canal invariante no tempo apresentado na Figura 6 , os algoritmos apresentam desempenhos semelhantes para valores de $\mathrm{Eb} / \mathrm{N} 0$ abaixo de $10 \mathrm{~dB}$, aproximadamente. Também de forma semelhante ao que mostra a Figura 6, vêse na Figura 10 que para valores de Eb/N0 entre 10 e $40 \mathrm{~dB}$ o desempenho do algoritmo LMS-BE praticamente não se altera, enquanto que o desempenho do KF-BE continua melhorando. O desempenho do algoritmo ASSLMS-BE fica aproximadamente inalterado entre 15 e $25 \mathrm{~dB}$, apresentando uma taxa de melhora do desempenho equivalente à do $\mathrm{KF}$ BE somente a partir dos $25 \mathrm{~dB}$. O comportamento observado para $E_{b} / N_{0}$ entre 15 e $25 \mathrm{~dB}$ pode ser explicado pelo mesmo motivo apresentado na análise dos resultados obtidos com canal invariante no tempo.

Na Figura 11 são apresentadas as curvas de taxa de erro para deslocamento Doppler máximo de $50 \mathrm{~Hz}$. Os algoritmos apresentam desempenhos próximos para valores de $E_{b} / N_{0}$ abaixo de aproximadamente $15 \mathrm{~dB}$. Até um valor de $E_{b} / N_{0}$ um pouco menor do que este, o LMS-BE apresenta os menores valores de BER. A partir dai, e até $E_{b} / N_{0}=40 \mathrm{~dB}$, o desempenho do algoritmo LMS-BE praticamente não se altera, enquanto que o do KF-BE continua melhorando. O desempenho do algoritmo ASSLMS-BE também continua melhorando, porém a taxas bem inferiores às do KF-BE, de modo que para os valores mais elevados da razão $E_{b} / N_{0} \mathrm{o}$ ASSLMS-BE mostra desempenho apenas um pouco melhor do que o do LMS-BE.

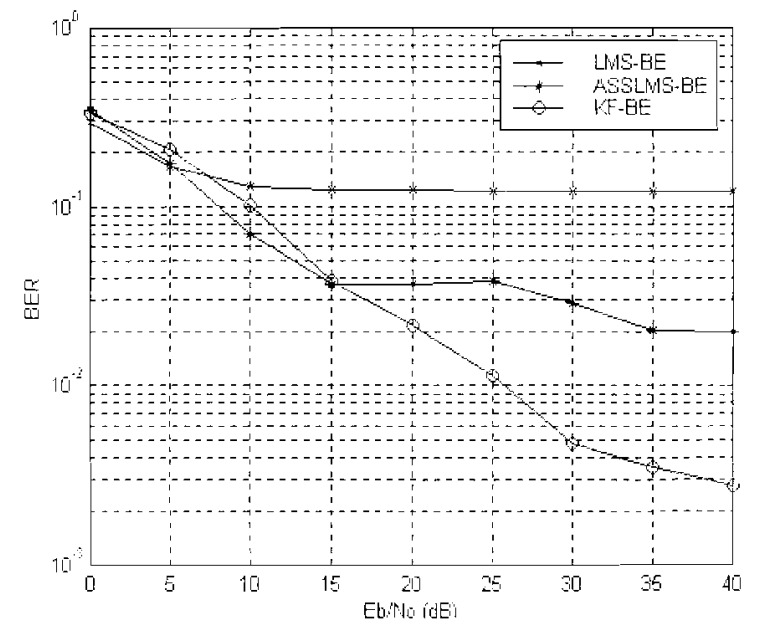

Figura 10. Desempenho de BER para $f_{D}=10 \mathrm{~Hz}$.

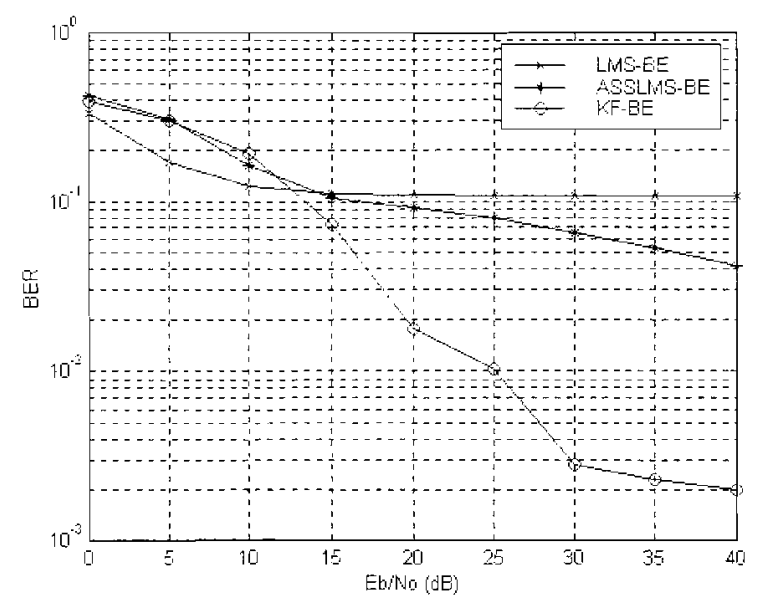

Figura 11. Desempenho de BER para $f_{D}=50 \mathrm{~Hz}$.

$\mathrm{Na}$ Figura 12 são apresentados os resultados de BER obtidos com $f_{D}=100 \mathrm{~Hz}$. Os algoritmos KF-BE e LMS-BE novamente apresentam desempenhos semelhantes para 
valores de $E_{b} / N_{0}$ abaixo de aproximadamente $15 \mathrm{~dB}$. A partir deste valor o desempenho do algoritmo LMS-BE praticamente não se altera, enquanto que o do KF-BE continua melhorando e praticamente se estabiliza para $E_{b} / N_{0}$ acima de $30 \mathrm{~dB}$. O desempenho do algoritmo ASSLMS-BE melhora de forma coerente com o aumento de $E_{b} / N_{0}$ até aproximadamente $30 \mathrm{~dB}$. A partir daí os valores de taxa de erro são atípicos, pois variam inversamente com a razão $E_{b} / N_{0}$

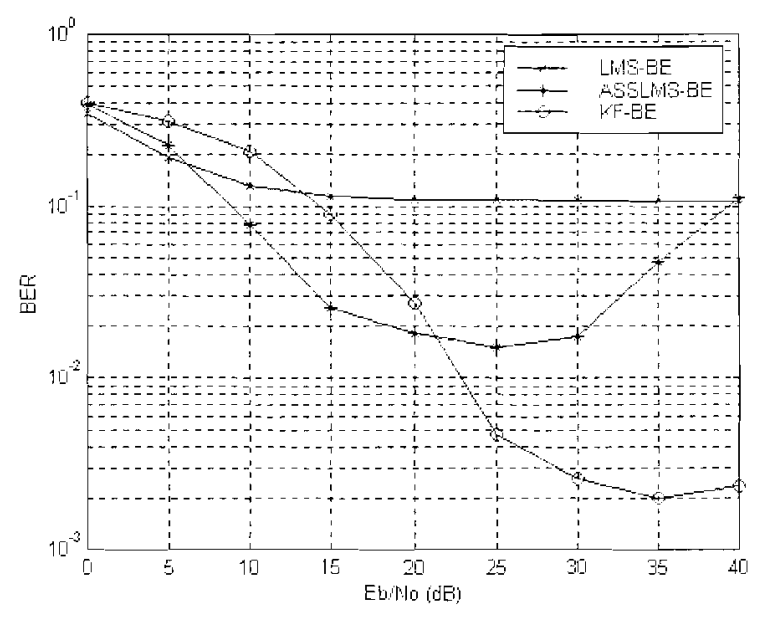

Figura 12. Desempenho de BER para $f_{D}=100 \mathrm{~Hz}$.

Estes resultados atípicos sugerem que o ajuste de parâmetros do ASSLMS-BE com base apenas na variância do ruído $\sigma_{n}^{2}$, como discutido na seção 3, é insuficiente para garantir um desempenho satisfatório em canais variantes com o tempo, o que provavelmente ser obtido considerando-se o efeito da velocidade de variação do canal na determinação destes parâmetros.

Como uma primeira tentativa de comprovar essa conjectura, fez-se modificações nos parâmetros deste algoritmo, aumentando o limiar de detecção cega de erro de equalização de 15 para 1000 , e também o limiar de detecção de instabilidade de 100 para 10000. Os resultados obtidos com essas modificações são apresentados na Figura $13 \mathrm{e}$ mostram melhora significativa de desempenho. Isto pode ser tomado como uma confirmação da argumentação acima e motiva uma investigação mais profunda para se obter uma metodologia de ajuste dos parâmetros do algoritmo ASSLMS-BE em função das características de variabilidade do canal com o tempo, o que será objeto de trabalho futuro.

A Figura 14 apresenta a evolução da porcentagem de bits errados por grupos de símbolos com $f_{D}=10 \mathrm{~Hz}$. Nota-se que o percentual de erros do equalizador LMS-BE durante a inicialização (primeiro grupo de bits) é muito grande, caindo lentamente com a evolução no tempo e voltando a crescer, também de forma lenta, a partir do processamento das 500 amostras iniciais. O KF-BE apresenta uma porcentagem de erros no primeiro grupo cerca de dez vezes inferior à do LMS-BE e também uma queda rápida com o tempo, conseguindo alcançar a situação de ausência de erros observados a partir do processamento de 800 amostras do sinal recebido. Já o ASSLMS-BE apresenta uma porcentagem de erros no primeiro grupo próxima da média entre a do LMS-BE e a do KF-BE, com queda mais acentuada do que a do KF-BE, alcançando a situação de ausência erros observados a partir de 700 amostras (antes do KF-BE, portanto).

A evolução da porcentagem de bits errados para $f_{D}=$ $50 \mathrm{~Hz}$ é apresentada na Figura 15. Novamente pode ser visto que a porcentagem de erros no primeiro grupo de símbolos detectados pelo equalizador LMS-BE é muito elevada (aproximadamente 20\%), sendo cerca da metade deste valor no caso do ASSLMS-BE e aproximadamente dez vezes menos para o KF-BE.

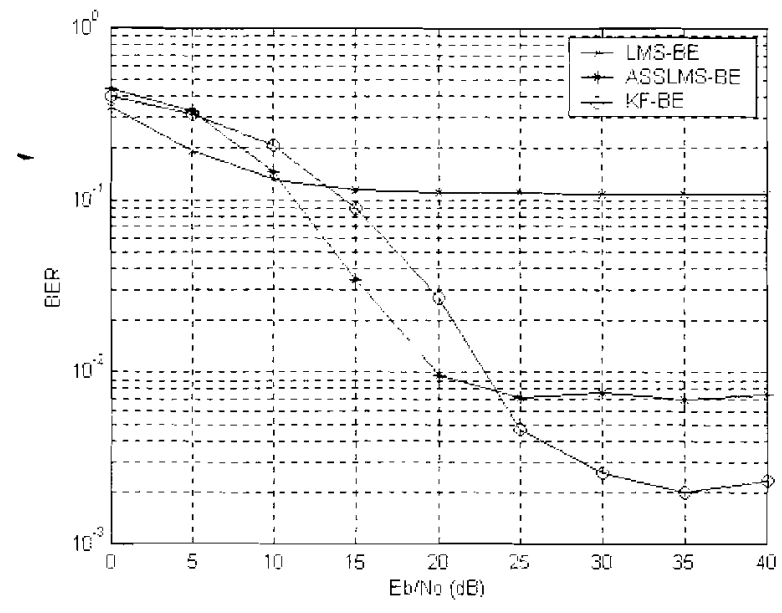

Figura 13. Desempenho de BER do equalizador ASSLMS-BE com novos parâmetros e $f_{D}=100 \mathrm{~Hz}$.

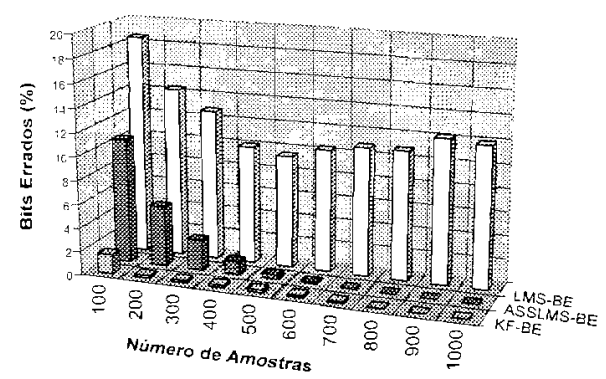

\begin{tabular}{lc|ccc:ccc|c|c|c|} 
& 100 & 200 & 300 & 400 & 500 & 600 & 700 & 800 & 900 & 1000 \\
\hline KF-BE & 1,636 & 0,212 & 0.188 & 0.208 & 0.2 & 0.184 & 0,128 & 0 & 0 & 0 \\
\hline ASSLMS-BEE & 10,572 & 5,132 & 2.592 & 1.104 & 0.356 & 0.124 & 0 & 0 & 0 & 0 \\
\hline LMS-BE & 18,616 & 14,396 & 12.812 & 10,08 & 9.552 & 10,38 & 10.892 & 10.904 & 12.18 & 11.996 \\
\hline
\end{tabular}

Figura 14. Porcentagem de bits errados para $f_{D}=10 \mathrm{~Hz}$ e $\mathrm{E}_{\mathrm{b}} / \mathrm{N}_{0}=40 \mathrm{~dB}$.

A Figura 15 também mostra que a porcentagem de erros cai ao longo dos blocos de símbolos para os três algoritmos, sendo mais significativa a queda produzida pelo KF-BE, seguida da obtida com o ASSLMS-BE. Com a evolução do processamento das amostras, o algoritmo KF-BE rapidamente alcança a situação de ausência de erros observados (depois das 300 amostras iniciais). Por outro lado, tanto para o LMS-BE quanto para o ASSLMS-BE, observa-se que a porcentagem de bits errados começa a crescer lentamente ao longo dos blocos de símbolos recebidos. Este fato indicam que esses algoritmos têm dificuldades no acompanhamento das variações do canal. 
Finalmente a Figura 16 mostra os resultados da evolução da porcentagem de bits errados obtidos com $f_{D}=100 \mathrm{~Hz}$. Desta vez, os resultados alcançados pelos algoritmos LMSBE e ASSLMS-BE são próximos do primeiro ao último grupo de símbolos. Ambos apresentam uma porcentagem de bits errados alta que pouco se altera ao longo do processamento. Já o algoritmo KF-BE continua apresentando uma baixa porcentagem de bits errados na inicialização e uma rápida convergência para situação de nenhum erro observado. Nota-se no entanto que, com a continuação do processamento, o KF-BE volta a apresentar erros, o que indica a ocorrência de dificuldade no acompanhamento das rápidas variações do canal com $f_{D}=$ $100 \mathrm{~Hz}$.

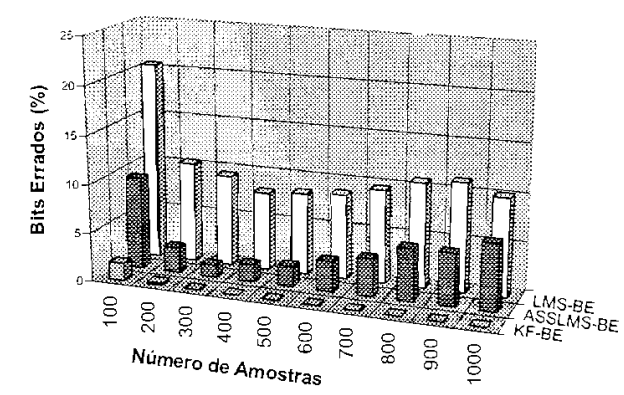

\begin{tabular}{lccc:c|cccccc} 
& 100 & 200 & 300 & 400 & 500 & 600 & 700 & 800 & 900 & 1000 \\
\hline KF-BE & 1,784 & 0,204 & 0 & 0 & 0 & 0 & 0 & 0 & 0 & 0 \\
\hline ASSLMS-BE & 9,536 & 2,592 & 1,216 & 1,744 & 2,108 & 3,096 & 3,884 & 5,392 & 5,424 & 6,828 \\
\hline LMS-BE & 20,516 & 10,38 & 9,464 & 8,084 & 8,428 & 8,736 & 9,62 & 10,804 & 11,236 & 10,136 \\
\hline
\end{tabular}

Figura 15. Porcentagem de bits errados para $f_{D}=50 \mathrm{~Hz}$ e $\mathrm{E}_{\mathrm{b}} / \mathrm{N}_{0}=40 \mathrm{~dB}$.

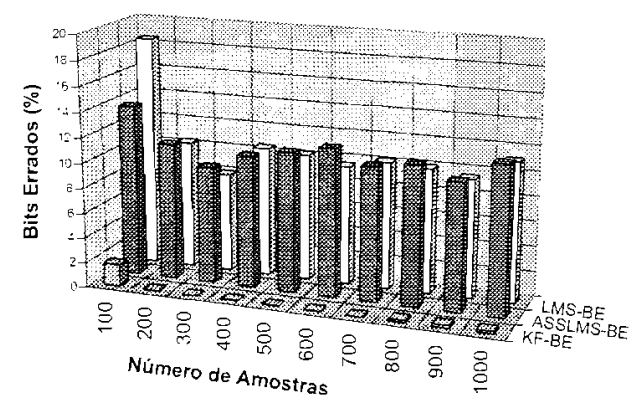

\begin{tabular}{lccc|c|cccccc} 
& 100 & 200 & 300 & 400 & 500 & 600 & 700 & 800 & 900 & 1000 \\
\hline KF-BE & 1,76 & 0 & 0 & 0,008 & 0 & 0 & 0 & 0,176 & 0,204 & 0.2 \\
\hline ASSLMS-BE & 13,72 & 10,944 & 9.384 & 10.576 & 11.2 & 11.784 & 10,676 & 11.144 & 10.104 & 11,788 \\
\hline LMS-BE & 18,488 & 10,304 & 7.968 & 10,428 & 10,184 & 9,512 & 10,152 & 9.976 & 9.468 & 11,1 \\
\hline
\end{tabular}

Figura 16. Porcentagem de bits errados para $f_{D}=100 \mathrm{~Hz}$ e $\mathrm{E}_{\mathrm{b}} / \mathrm{N}_{0}=40 \mathrm{~dB}$.

A provável causa deste fato se deve ao desajuste entre o modelo de passeio aleatório usado na equação de estado e o modelo efetivo de canal empregado. com espectro Doppler de Jakes. Conforme verificado em [14] para alguns esquemas com treinamento, este tipo de desajuste pode causar perdas significativas no desempenho de receptores adaptativos baseados em filtragem de Kalman, em canais com desvanecimento rápido. Também se verificou em [14] que o desempenho deste tipo de receptor pode ser radicalmente modificado para melhor empregando-se modelagem autoregressiva de segunda ordem na equação de estado. Apesar das diferenças entre os receptores considerados em [14] e o esquema KF-BE, parece razoável esperar que o desempenho deste também melhore com a utilização de modelagem mais adequada na equação de estado.

A Figura 17 apresenta os resultados de MSE na estimação da RIC obtidos $\operatorname{com} f_{D}=10 \mathrm{~Hz}$ e $E b / N_{0}=40 \mathrm{~dB}$. Nesta Figura pode ser visto que, apesar do algoritmo KF-BE apresentar a taxa de convergência mais rápida, o menor valor de MSE é alcançado pelo ASSLMS-BE, que também exibe taxa de convergência rápida. Já o LMS-BE, além de mostrar convergência lenta, produz um elevado valor de MSE residual. Nota-se ainda que a curva de MSE na estimação da RIC do algoritmo ASSLMS-BE fica abaixo das curvas obtidas com os outros dois algoritmos a partir da amostra 170, aproximadamente, ficando muito próxima da produzida pelo KF-BE.

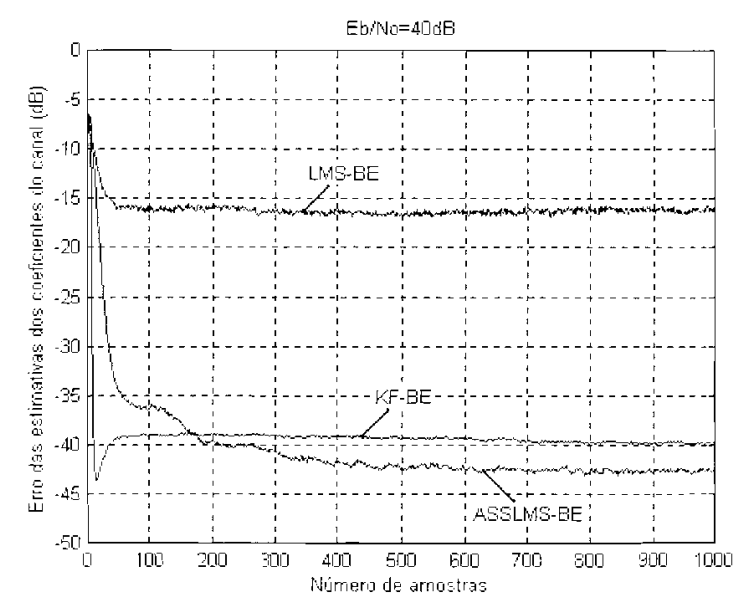

Figura 17. Trajetória de EMQ na estimação dos coeficientes do canal para $f_{D}=10 \mathrm{~Hz}$ e $\mathrm{E}_{\mathrm{b}} / \mathrm{N}_{0}=40 \mathrm{~dB}$.

Um comportamento que se destaca na Figura 17 é a ocorrência de um undershoot inicial na curva de MSE do algoritmo KF-BE. Esse comportamento também pode ser creditado ao desajuste entre a equação de estado admitida pelo KF-BE e o modelo efetivo de variação do canal com o tempo. Cabe notar que os filtros de Kalman utilizados neste equalizador são inicializados com base no conhecimento "a priori" do canal. Desta forma, na sua inicialização o KF-BE está ajustado ao modelo de canal e por isso o EMQ caj significativamente com o processamento da primeira amostra do sinal recebido. No entanto, há um aumento do EMQ logo em seguida, devido ao fato de que o modelo de passeio aleatório usado na equação de estado não representa adequadamente a evolução dos coeficientes da RIC com o tempo.

Uma confirmação simples deste argumento pode ser vista nas curvas de MSE na estimação do canal por filtragem de Kalman apresentadas na Figura 18, que foram obtidas admitindo-se conhecidos os símbolos transmitidos, e considerando duas situações distintas no que diz respeito à equação de estado. Numa delas o modelo de estado (AR-1) está perfeitamente ajustado ao do canal. Na outra usa-se um modelo de passeio aleatório na equação de estado, enquanto o canal segue o modelo descrito acima, com espalhamento 
Doppler de Jakes. Nota-se na Figura 18 que o ajuste entre o modelo de canal e o utilizado na equação de estado produz a eliminação do "undershoot", além de levar a valores de MSE residuais mais baixos. Um comportamento bem diferente, e a ocorrência de undershoot inicial, em particular, é observado na mesma Figura quando se emprega o modelo de passeio aleatório em desajuste como modelo de canal.

A Figura 18 também mostra que a curva de MSE obtida com modelo desajustado é mais suave do que a obtida com modelo ajustado. Isto se deve à diferença na velocidade de variação de cada canal simulado, e ao uso do mesmo número de realizações estimação do EMQ nos dois casos. Em termos mais específicos, o canal ajustado é de fato bem mais "rápido" do que o canal do ajustado (a variância do modelo AR-1 é relativamente alta), o que leva à necessidade de um número maior de realizações independentes para obter uma estimativa de MSE mais suave.

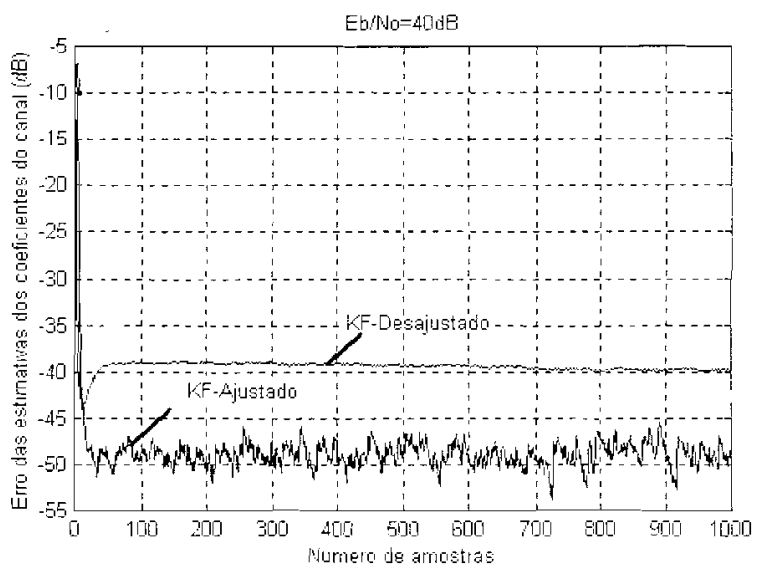

Figura 18. EMQ produzido pelo filtro de Kalman na estimação dos coeficientes do canal com símbolos conhecidos, sob duas condições distintas de ajuste entre o modelo de estado e o de canal.

Os resultados de MSE na estimaçào da RIC para $f_{D}=50$ $\mathrm{Hz}$ e $E b / N_{0}=40 \mathrm{~dB}$ são mostrados na Figura 19. Nota-se que o algoritmo KF-BE apresenta a taxa de convergência mais rápida, e também o menor valor de MSE. O ASSLMS$\mathrm{BE}$ apresenta inicialmente uma queda mais rápida no MSE do que o LMS-BE (porém não comparável à do KF-BE), alcançando um valor de MSE intermediário entre os alcançados pelos outros dois esquemas. No entanto, a partir da amostra 200, aproximadamente, o MSE produzido pelo ASSLMS-BE começa a aumentar até alcançar a mesma faixa de valores produzida pelo LMS-BE.

A Figura 20 mostra os resultados de MSE na estimaçào do canal $\operatorname{com} f_{D}=100 \mathrm{~Hz}$ e $E b / N_{0}=40 \mathrm{~dB}$. O algoritmo KF$\mathrm{BE}$ apresenta a taxa de convergência mais rápida e valores muito baixos de MSE residual. Os comportamentos dos algoritmos LMS-BE e ASSLMS-BE são muito semelhantes, tanto no que se refere à taxa de convergência quanto ao valor do MSE residual.

De uma forma geral, os resultados desta sub-seção destacam mais uma vez o desempenho muito superior do esquema KF-BE em relação ao do esquema LMS-BE, para todos os valores utilizados de deslocamento Doppler máximo $(10,50$ e $100 \mathrm{~Hz})$ tanto no que se refere à BER estimada quanto no que diz respeito aos valores de MSE.

Considerando o algoritmo ASSLMS-BE, os resultados para o deslocamento Doppler máximo de $10 \mathrm{~Hz}$ mostraram que a adoçào do passo adaptativo levou a melhoramentos significativos na taxa de convergència e no MSE residual das estimativas do canal. assim como na taxa de erro de bit. De forma semelhante ao caso do canal invariante no tempo, obteve-se também com $f_{D}=10 \mathrm{~Hz}$ características de desempenho do ASSLMS-BE intermediárias entre as produzidas pelo LMS-BE e pelo KF-BE, ficando o desempenho mais próximo do produzido pelo segundo.

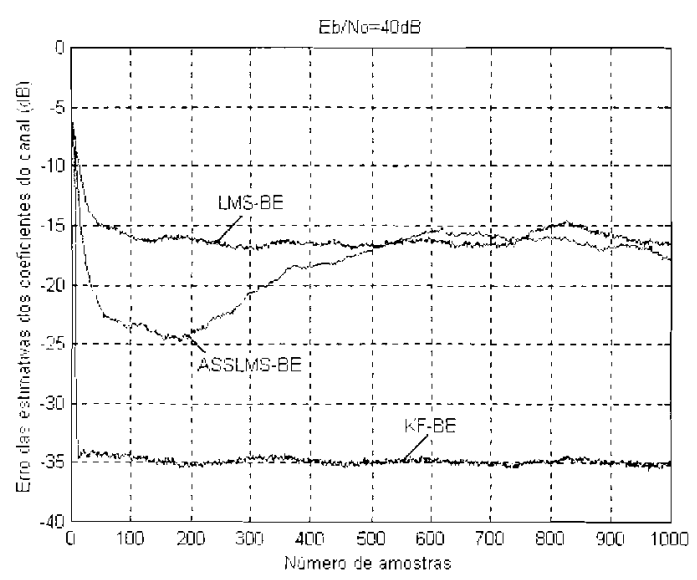

Figura 19. Trajetória de EMQ na estimação dos coeficientes do canal para $f_{D}=50 \mathrm{~Hz}$ e $\mathrm{E}_{\mathrm{b}} / \mathrm{N}_{0}=40 \mathrm{~dB}$.

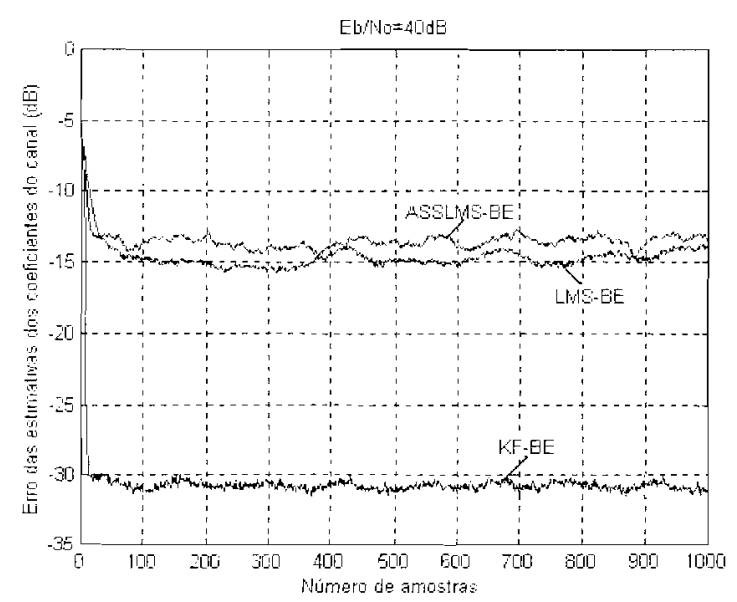

Figura 20. Trajetória de EMQ na estimação dos coeficientes para $f_{D}=100 \mathrm{~Hz}$ e $\mathrm{E}_{\mathrm{b}} / \mathrm{N}_{0}=40 \mathrm{~dB}$.

Já os resultados obtidos com deslocamentos Doppler máximos de $50 \mathrm{~Hz}$ e $100 \mathrm{~Hz}$ mostraram a incapacidade do esquema ASSLMS-BE (na forma como foi implementado) de acompanhar variaçôes mais rápidas do canal, indicando a necessidade de um novo procedimento de ajuste dos valores de seus parâmetros, levando em conta a velocidade de variação do canal, o que foi confirmado $\mathrm{em}$ um teste simples. Mesmo assim, observou-se que para $f_{D}=50 \mathrm{~Hz}$, o equalizador ASSLMS-BE ainda consegue produzir desempenho de taxa de erro superior ao esquema LMS-BE. 


\section{CONCLUSÕES}

Um novo algoritmo LMS de passo adaptativo foi aqui introduzido para utilização numa estrutura Bayesiana de equalização cega apresentada em [1]. O ajuste de passo aqui proposto se vale de uma métrica desenvolvida em [8] para uso em um algoritmo de detecção cega de erros de equalização. Simulou-se o novo esquema de equalização assim obtido, considerando um modelo de canal com desvanecimento seletivo em freqüência e diversas atribuições de parâmetros deste modelo, particularmente no que diz respeito à variabilidade da resposta ao impulso do canal com o tempo. Para efeito de comparação, também se avaliou nas mesmas condições o desempenho dos esquemas originalmente propostos em [1], que se diferenciam pela utilização do LMS padrão e do filtro de Kalman para estimação do canal.

Os resultados obtidos mostraram que a adoção do algoritmo LMS de passo adaptativo levou a melhoramentos significativos tanto no que se refere à taxa de convergência e ao MSE residual das estimativas do canal, quanto à taxa de erro de bit, principalmente para SNR mais altas. Obtiveram-se assim. caracteristicas de desempenho e complexidade intermediárias em relação às dos esquemas LMS-BE e a KF-BE. Em termos mais específicos, a complexidade ficou mais próxima da do equalizador com algoritmo LMS padrão, e o desempenho, para canal invariante ou lentamente variante com o tempo, ficou bem mais próximo do produzido pelo esquema com filtros de Kalman. Em casos de variação mais rápida da resposta ao impulso do canal, observou-se significativas degradações no desempenho do esquema aqui proposto e a necessidade de se reajustar os seus parâmetros, levando em conta a velocidade de variação do canal.

Como propostas para futuros trabalhos decorrentes deste, colocam-se as seguintes:

- Investigação da relação entre a velocidade de variação do canal e os parâmetros do equalizador ASSLMS-BE buscando uma nova estratégia de ajuste de passo do LMS que leve em conta esta relação;

- Investigação do ajuste de parâmetros e do desempenho do equalizador ASSLMS-BE para o caso de modulação QPSK:

- Avaliação da robustez do desempenho dos esquemas aqui investigados a desajuste maiores entre o modelo suposto pelo equalizador e o modelo físico de canal.

\section{REFERÊNCIAS}

[1] R. A. ILTIS, J. J. SHYNK, K. GIRIDHAR, "Bayesian Algorithms for Blind Equalization Using Parallel Adaptive Filtering", IEEE Trans. on Comm. March 1994.

[2] K. ABEND AND B. D. FRITCHMAN, "Statistical detection for communication channels with intersymbol interference", Proc. IEEE, VOL.58, pp. 779-785, May 1970.

[3] A. G. MELO, E. L. PINTO, "Performance Evaluation of Bayesian Blind Equalizers Over WSS-US Channels", XVII SBT. 1999.

[4] J. B. EVANS. P. XUE, B. LIU, "Analysis and Implementation of Variable Step Size Algorithms", IEEE Transactions on Signal Processing, August 1993, pp. $2517-$ 2535 .
[5] R. H. KWONG, E. W. JOHNSON, "A Variable Step Size LMS Algorithm", IEE Transaction on Signal Processing, vol. 40, pp. 1633-1642, July 1992.

[6] V. J. MATHEUS, Z. XIE, "A Stochastic Gradient Adaptive Filter with Gradient Adaptive Step Size", IEEE Transaction on Signal Processing, vol.41, pp.2075-2087, June 1993.

[7] K. MAYAS, T.ABOULNASR, "A Robust Variable Step Size LMS-type Algorithm: Analysis and Simulation", in Proc. ICASSP 95, pp.1408-1411, 1995.

[8] K. DOGANÇAY, V. KRISHNAMURTHY, "Blind On-Line Testing for Equalization Errors in Digital Communication Systems", IEEE Trans. on Information Theory, vol.44, no. 4 . pp. 1677-1686, July 1998.

[9] K. DOGANÇAY, R. A. KENNEDY, "Blind detection of equalization errors in communication systems", IEEE Trans. on Information Theory, vol.43, pp. 469-482, March 1997.

[10] N. AL-DHAHIR, "A Computationally Efficient FIR MMSEDFE for CCI-Impaired Dispersive Channels", IEEE Transactions on Signal Processing, vol.45. no.1, January 1997.

[11] MOTOROLA, Inc., DSP 56000 Digital Signal Processor User's Manual, 1986.

[12] EIA/TIA IS-136; $800 \mathrm{MHz}$ TDMA Cellular - Radio Interface - Mobile Station - Base Station Compatibility - Digital Control Channel, December, 1994.

[13] A. G. GUIMARÃES, "Modelagem e Simulação de Canais Rádio Móveis", Tese de Mestrado, IME, 1998.

[14] E. L. PINTO, J. F. GALDINO, "A Simulation Study of Adaptive Filtering Applied to MLSE-PSP Receivers", Proceedings of the I998 IEEE Military Communications Conference (MILCOM'98) - Unclassified Program, Bedford, MA, EEUU, 1998

Alexandre Guedes de Melo é graduado em Engenharia Elétrica pelo Instituto Militar de Engenharia (IME), em 1994, tem mestrado na área de sistemas de comunicações também pelo IME, concluido em 2000. Suas principais áreas de pesquisa são: equalizaçâo cega e avaliação de desempenho de sistemas de transmissào digital. Desde 1999, trabalha na Motorola Industrial do Brasil como Engenheiro de Desenvolvimento de Novos Produtos para o Setor de Comunicaçōes Pessoais.

Ernesto Leite Pinto é graduado em Engenharia Elétrica pela Universidade Federal da Paraíba, em 1983, tem mestrado e doutorado na área de sistemas de comunicações pela PUC/RJ, concluídos em 1986 e 1998, respectivamente. É professor adjunto IV do Departamento de Engenharia Elétrica do IME, onde trabalha desde 1987. Suas principais áreas de pesquisa são: avaliação de desempenho de sistemas de transmissão digital e processamento digital de sinais aplicados a sistemas de comunicações digitais. É autor de diversos trabalhos publicados em anais de congressos e revistas de sociedades científicas nacionais e intenacionais. É atualmente membro do Conselho Deliberativo da Sociedade Brasileira de Telecomunicações. 